TRANSACTIONS OF THE

AMERICAN MATHEMATICAL SOCIETY

Volume 357, Number 10, Pages 3831-3863

S 0002-9947(05)03945-0

Article electronically published on May 10, 2005

\title{
STRONG CHIP, NORMALITY, AND LINEAR REGULARITY OF CONVEX SETS
}

\author{
ANDREW BAKAN, FRANK DEUTSCH, AND WU LI
}

\begin{abstract}
We extend the property $(\mathrm{N})$ introduced by Jameson for closed convex cones to the normal property for a finite collection of convex sets in a Hilbert space. Variations of the normal property, such as the weak normal property and the uniform normal property, are also introduced. A dual form of the normal property is derived. When applied to closed convex cones, the dual normal property is the property $(\mathrm{G})$ introduced by Jameson. Normality of convex sets provides a new perspective on the relationship between the strong conical hull intersection property (strong CHIP) and various regularity properties. In particular, we prove that the weak normal property is a dual characterization of the strong CHIP, and the uniform normal property is a characterization of the linear regularity. Moreover, the linear regularity is equivalent to the fact that the normality constant for feasible direction cones of the convex sets at $x$ is bounded away from 0 uniformly over all points in the intersection of these convex sets.
\end{abstract}

\section{INTRODUCTION}

Briefly, this paper studies three forms of properties that can be imposed on a finite collection of closed convex sets in a Hilbert space: the strong CHIP, normality, and linear regularity.

The concept of CHIP was first introduced by Chui, Deutsch, and Ward [12] as a sufficient condition for an unconstrained reformulation of a constrained best approximation problem. Afterward, Deutsch, Li, and Ward [18] found that a stronger version of CHIP was actually needed for the reformulation of the constrained best approximation. In special cases, the reformulation leads to an unconstrained reformulation of a constrained optimization problem, which allows one to use various unconstrained optimization algorithms to solve constrained minimization problems [18. Later, Deutsch [15] showed that the strong CHIP is a geometric version of the basic constraint qualification for constrained optimization problems.

The concept of linear regularity was first introduced by Bauschke and Borwein as a key condition in establishing a linear convergence rate of iterates generated by the cyclic projection algorithm for finding the projection from a point to an intersection of finitely many closed convex sets $C_{1}, \ldots, C_{m}$ [5, 4, 6, 8, 7]. Later, Bauschke,

Received by the editors May 30, 2002.

2000 Mathematics Subject Classification. Primary 90C25, 41A65; Secondary 52A15, 52A20, $41 \mathrm{~A} 29$.

Key words and phrases. Moreau-Rockafellar equality, Jameson's property (N), Jameson's property $(\mathrm{G})$, the conical hull intersection property (the CHIP), the strong conical hull intersection property (the strong CHIP), basic constraint qualification, linear regularity, bounded linear regularity, normal property, weak normal property, uniform normal property, dual normal property. 
Borwein, and $\mathrm{Li}\left[9\right.$ proved that the bounded linear regularity of $\left\{C_{1}, \ldots, C_{m}\right\}$ implies the strong CHIP of $\left\{C_{1}, \ldots, C_{m}\right\}$. But the converse is not true (see [3, 10]). Linear regularity is also related to error estimates for approximate solutions of a feasibility problem (such as Hoffman's error bounds) [10, 30, 25].

The normal property for two convex cones was introduced by Jameson [26] to study the closedness of the sum of their dual cones. The dual form of the normal property (called property $(G)$ by Jameson) is actually closely related to linear regularity for two closed convex cones (see [26, Theorem 2.1] or [9] Theorem 6.5]).

Since the strong CHIP and linear regularity are properties for a finite collection of closed convex sets, a natural question is whether it is meaningful to extend the definition of Jameson's normal property for a collection of convex sets. The main purpose of this paper is to show that such an extension does provide new perspectives on the relationship between the strong CHIP and linear regularity. By using various normal properties, we obtain new characterizations of the strong CHIP and linear regularity.

Even in a finite-dimensional space setting, our results are new. Readers may read the paper under the assumption that $H$ is a finite-dimensional Euclidean space. This will help readers understand the main ideas without having to deal with topological concepts in a Hilbert space (such as weak convergence, etc.).

The paper is organized as follows. In Section 2 we list a few facts about polars of convex sets and some algebraic/topological properties about convex sets. All results in Section 2 are needed in the proofs of the main results and should be studied only if the reader has difficulty in understanding the proofs in the following sections. In Section 3 various normal properties are introduced and a hierarchy among various normal properties is established. We devote Section 4 to the dual normality and show that the dual normality property is a generalization of Jameson's property $(\mathrm{G})$ for closed convex cones. In Section 5 we prove that the weak normal property is a dual characterization of the strong conical hull intersection property. Finally, various characterizations of the linear regularity are given in Section 6. In particular, we prove that linear regularity is equivalent to the uniform normal property. Moreover, a collection of closed convex sets $\left\{C_{1}, \ldots, C_{m}\right\}$ is linearly regular if and only if the normality constant for the collection of feasible direction cones $\left\{\operatorname{con}\left(C_{1}-x\right), \ldots, \operatorname{con}\left(C_{m}-x\right)\right\}$ is bounded away from 0 uniformly over all $x \in \bigcap_{1}^{m} C_{i}$.

We conclude this Introduction by listing the notations that will be used throughout the paper. Unless otherwise specified, $H$ will always denote a (real) Hilbert space with inner product $\langle x, y\rangle$, norm $\|x\|=\sqrt{\langle x, x\rangle}$, and unit ball $B_{H}:=\{x \in$ $H \mid\|x\| \leq 1\}$. An important example of a Hilbert space is a $k$-dimensional Euclidean space $\mathbb{R}^{k}$, which is the vector space of all $k$-tuples $x=(x(1), \ldots, x(k))$ of real numbers $x(i)$ with the inner product $\langle x, y\rangle=\sum_{1}^{k} x(i) y(i)$.

For a given subset $S$ of $H$, we denote the closure, interior, and relative interior of $S$ by $\bar{S}$, $\operatorname{int}(S)$, and $\operatorname{ri}(S)$, respectively. In addition, the convex hull and the affine span of $S$ are denoted by $\operatorname{co}(S)$ and aff $(S)$, respectively. A convex cone is a subset $K$ of $H$ with the property that $K+K \subset K$ and $\theta K \subset K$ for all $\theta \geq 0$. The conical hull of $S$, denoted by $\operatorname{con}(S)$, is the intersection of all convex cones that contain $S$; hence $\operatorname{con}(S)$ is the smallest convex cone that contains $S$. The closures of $\operatorname{co}(S)$ and $\operatorname{con}(S)$ are abbreviated by $\overline{\mathrm{co}}(S)$ and $\overline{\mathrm{con}}(S)$, respectively. The polar of $S$ is the set $S^{\circ}:=\{x \in H \mid\langle x, y\rangle \leq 1$ for every $y \in S\}$; this is a closed convex set that 
contains the origin. The distance from a point $x \in H$ to the set $S$ is defined by $d(x, S):=\inf _{y \in S}\|x-y\|$.

There are a number of convex cones that one can generate from a given convex subset $C$ of $H$. We list those that are of main importance to this work. The dual cone (or negative polar) of $C$ is the set $C^{\ominus}:=\{x \in H \mid\langle x, y\rangle \leq 0$ for every $y \in C\}$. Note that the dual cone of $C$ is a closed convex cone; and if $C$ is a convex cone, then $C^{\ominus}=C^{\circ}$. The orthogonal complement of $C$ is the set $C^{\perp}:=\{x \in H \mid$ $\langle x, y\rangle=0$ for every $y \in C\}$. If $C$ is a linear subspace of $H$, then $C^{\circ}=C^{\ominus}=C^{\perp}$. The normal cone (respectively, tangent cone) to $C$ at a point $x \in H$ is the set $N_{C}(x)=(C-x)^{\ominus}$ (respectively, $T_{C}(x)=\overline{\operatorname{con}}(C-x)$ ). It is not hard to show that $N_{C}(x)^{\circ}=N_{C}(x)^{\ominus}=T_{C}(x)$ and $T_{C}(x)^{\circ}=T_{C}(x)^{\ominus}=N_{C}(x)$. That is, $T_{C}(x)$ and $N_{C}(x)$ are the dual cones (and polars) of each other. The cone of feasible directions (see [22 p. 135]) to $C$ at a point $x \in C$ is the set $\operatorname{con}(C-x)$. The recession cone of $C$ is the set

$$
0^{+} C:=\{x \in H \mid y+\theta x \in C \text { for all } \theta \geq 0 \text { and } y \in C\} .
$$

Alternatively, $0^{+} C=\{x \in H \mid C+x \subset C\}$. From this it is easy to see that (i) $0^{+} C$ is a convex cone, (ii) $0^{+} C=C$ if and only if $C$ is a convex cone, and (iii) if $C$ is a closed convex set, then $0^{+} C$ is a closed convex cone. It is also not difficult to verify that

$$
0^{+} C^{\circ}=C^{\ominus}
$$

and for arbitrary closed convex subsets $C_{i}$ of $H$ (see [29]),

$$
0^{+}\left(\bigcap_{i=1}^{m} C_{i}\right)=\bigcap_{i=1}^{m} 0^{+} C_{i} .
$$

In this paper, we also need some topological concepts in Hilbert spaces. Recall that a sequence $\left\{x_{n}\right\}$ in $H$ converges weakly to $x^{*}$ if

$$
\lim _{n \rightarrow \infty}\left\langle x_{n}, y\right\rangle=\left\langle x^{*}, y\right\rangle \text { for all } y \in H .
$$

A subset $D$ of $H$ is said to be weakly closed, if $D$ contains the limit of any weakly convergent sequence $x_{n} \in D$. A subset $D$ of $H$ is said to be weakly compact, if for any sequence $x_{n} \in D$, there exists a subsequence of $\left\{x_{n}\right\}$ that converges weakly to a point in $D$. When $H=\mathbb{R}^{k}, x_{n}$ converges weakly to $x$ if and only if $\lim _{n \rightarrow \infty}\left\|x_{n}-x\right\|=0$; and a set $D$ is weakly compact if and only if $D$ is closed and bounded.

\section{Properties of Convex Sets}

In this section, we collect some facts about polars and convex/conical hulls in Lemma 2.1. Moreover, we will give a few technical lemmas about algebraic and topological properties of convex sets. These lemmas contain some technical statements needed later. This will make it easier to follow the main ideas in the proofs of the results concerning strong CHIP, normality, and linear regularity in the following sections. Readers should study this section only if they have difficulty in understanding the proofs given in Sections 3-6.

Lemma 2.1(1)-(8) contain a few facts about polars, and some properties concerning convex/conical hull are given in Lemma 2.1(9)-(11). Most of these statements 
can be found in a standard convex analysis textbook (cf. [34, 22] or [16, sections 4.4-4.6]). However, interested readers can also derive them from the definitions.

Lemma 2.1. Let $C, C_{i}$ be convex sets and let $K, K_{i}$ be convex cones in $H$.

(1) $C^{\circ}$ is a closed convex set that contains 0 and $C^{\circ}=(\bar{C})^{\circ}$.

(2) If $C_{1} \subset C_{2}$, then $C_{1}^{\circ} \supset C_{2}^{\circ}$.

(3) $(\alpha C)^{\circ}=\frac{1}{\alpha} C^{\circ}$ for each constant $\alpha \neq 0$.

(4) (Bipolar theorem) $C^{\circ \circ}=\overline{\mathrm{co}}(C \cup\{0\})$. In particular, if $0 \in C$, then $C^{\circ \circ}=$ $\bar{C}$.

(5) $K^{\circ}=K^{\ominus}$ and $\left(\bigcap_{1}^{m} K_{i}\right)^{\circ} \supset \overline{\sum_{1}^{m} K_{i}^{\circ}}$. Moreover, if $K_{i}$ are closed, then $\left(\cap_{1}^{m} K_{i}\right)^{\circ}=\overline{\sum_{1}^{m} K_{i}^{\circ}}$.

(6) $\left(\bigcup_{1}^{m} C_{i}\right)^{\circ}=\left[\operatorname{co}\left(\bigcup_{1}^{m} C_{i}\right)\right]^{\circ}=\bigcap_{1}^{m} C_{i}^{\circ}$.

(7) If $C_{i}$ are closed and $0 \in \bigcap_{1}^{m} C_{i}$, then $\left(\bigcap_{1}^{m} C_{i}\right)^{\circ}=\overline{\mathrm{co}}\left(\bigcup_{1}^{m} C_{i}^{\circ}\right)$.

(8) If $0 \in C$, then $(K+C)^{\circ}=K^{\circ} \cap C^{\circ}$.

(9) If $0 \in \bigcap_{1}^{m} C_{i}$, then $\operatorname{co}\left(\bigcup_{1}^{m} C_{i}\right) \subset \sum_{1}^{m} C_{i} \subset m \cdot \operatorname{co}\left(\bigcup_{1}^{m} C_{i}\right)$ and $\operatorname{co}\left(\bigcup_{1}^{m} K_{i}\right)=$ $\sum_{1}^{m} K_{i}$.

(10) $\operatorname{con}(C)=\{\theta x \mid \theta \geq 0, x \in C\}=\bigcup_{\theta \geq 0}(\theta C)$; if $0 \in C$, then $\operatorname{con}(C)=$ $\bigcup_{\theta>0}(\theta C)$.

(11) If $C$ is weakly compact, then $\overline{\mathrm{co}}(C)=\operatorname{co}(C)$.

Remark 2.1. In the sequel, we will use the following simple fact without explicit mention:

If $D$ is a nonempty set and $K$ is a convex cone in $H$, then

$$
\bar{D}=\bigcap_{\varepsilon>0}\left(D+\varepsilon B_{H}\right) \quad \text { and } \quad \theta(K+D)=K+\theta D \quad \text { for every } \theta>0 .
$$

The following lemma shows that in a certain case, we can exchange the order of intersection and union for convex sets.

Lemma 2.2. Let $A_{i}(\theta)$ be a set for each $\theta>0$ and $i \in\{1,2, \ldots, m\}$. Suppose that this collection of sets is monotone. (That is, either $A_{i}\left(\theta_{1}\right) \subset A_{i}\left(\theta_{2}\right)$ for each $i$ whenever $0<\theta_{1}<\theta_{2}$, or $A_{i}\left(\theta_{1}\right) \supset A_{i}\left(\theta_{2}\right)$ for each $i$ whenever $0<\theta_{1}<\theta_{2}$.) Then

$$
\bigcap_{i=1}^{m}\left[\bigcup_{\theta>0} A_{i}(\theta)\right]=\bigcup_{\theta>0}\left[\bigcap_{i=1}^{m} A_{i}(\theta)\right] \text {. }
$$

In other words, the union (over all $\theta>0$ ) commutes with the intersection (over all $i \in\{1,2, \ldots, m\})$.

Proof. We assume the sets are monotonely increasing. The proof for the decreasing case is similar. Let $A=\bigcup_{\theta>0}\left(\bigcap_{i=1}^{m} A_{i}(\theta)\right)$ and $B=\bigcap_{i=1}^{m}\left(\bigcup_{\theta>0} A_{i}(\theta)\right)$. We must show that $A=B$. If $x \in A$, then there exists $\theta>0$ such that $x \in \bigcap_{i=1}^{m} A_{i}(\theta)$. It follows that $x \in A_{i}(\theta)$ for each $i$, which implies that $x \in \bigcup_{\theta>0} A_{i}(\theta)$ for each $i$. Hence $x \in \bigcap_{i=1}^{m}\left(\bigcup_{\theta>0} A_{i}(\theta)\right)=B$. Thus $A \subset B$.

Conversely, if $x \in B$, then $x \in \bigcup_{\theta>0} A_{i}(\theta)$ for each $i$, which implies that for each $i$ there exists $\theta_{i}>0$ such that $x \in A_{i}\left(\theta_{i}\right)$. Let $\theta=\max _{1 \leq i \leq n} \theta_{i}$. Since the $A_{i}$ 's are increasing, we have that $x \in A_{i}(\theta)$ for all $i$, which implies that $x \in \bigcap_{1}^{m} A_{i}(\theta)$, and hence $x \in A$. Thus $B \subset A$, and the proof is complete. 
The next lemma is about the closure and closedness of convex sets.

Lemma 2.3. Suppose that $C_{1}$ and $C_{2}$ are convex subsets of $H$.

(1) If $C_{1}, C_{2}$ are closed and $C_{1}$ is bounded, then $C_{1}+C_{2}$ is also closed.

(2) If $C_{2} \cap \operatorname{int}\left(C_{1}\right) \neq \emptyset$, then $\overline{C_{1} \cap C_{2}}=\overline{C_{1}} \cap \overline{C_{2}}$.

(3) $\overline{C_{1}}+\overline{C_{2}} \subset \overline{\overline{C_{1}}+\overline{C_{2}}}=\overline{C_{1}+C_{2}}$.

Proof. (1) Assume that $C_{1}$ and $C_{2}$ are closed. Let $z_{n}=x_{n}+y_{n} \in C_{1}+C_{2}$ with $\lim _{n} z_{n}=z \in H$. Since $C_{1}$ is a bounded closed convex set, it is weakly compact. We may assume (by replacing $x_{n}$ by a convergent subsequence) that $x_{n}$ converges weakly to $x \in C_{1}$. Then $y_{n}=z_{n}-x_{n}$ converges weakly to $z-x$. Since $C_{2}$ is a closed convex set, it is weakly closed. Thus, $z-x \in C_{2}$ and $z=x+(z-x) \in C_{1}+C_{2}$. This proves the closedness of $C_{1}+C_{2}$.

(2) It suffices to prove that $\overline{C_{1}} \cap \overline{C_{2}} \subset \overline{C_{1} \cap C_{2}}$. Let $x \in \overline{C_{1}} \cap \overline{C_{2}}$. Then there exist $x_{n} \in C_{2}$ such that $\lim _{n} x_{n}=x$. Since $C_{2} \cap \operatorname{int}\left(C_{1}\right) \neq \emptyset$, there exist $x_{0} \in C_{1} \cap C_{2}$ and $\varepsilon>0$ such that $x_{0}+2 \varepsilon B_{H} \subset C_{1}$. Then $(1-t) x+t\left(x_{0}+2 \varepsilon B_{H}\right) \subset \overline{C_{1}}$ for $0<t<1$, which implies that

$$
(1-t) x+t\left(x_{0}+\varepsilon B_{H}\right) \subset \operatorname{int}\left(\overline{C_{1}}\right)=\operatorname{int}\left(C_{1}\right) \text { for all } 0<t<1 .
$$

Fix $t$ for the moment. Then, for sufficiently large $n$, we have $\left\|x-x_{n}\right\| \leq t \varepsilon$. Therefore,

$$
(1-t) x_{n}+t x_{0} \in(1-t) x+t\left(x_{0}+\varepsilon B_{H}\right) \subset C_{1} .
$$

Since $x_{0}, x_{n} \in C_{2}$, we have $(1-t) x_{n}+t x_{0} \in C_{1} \cap C_{2}$ for sufficiently large $n$. Letting $n \rightarrow \infty$, we obtain $(1-t) x+t x_{0} \in \overline{C_{1} \cap C_{2}}$ for $0<t<1$. Taking the limit with respect to $t$ as $t \rightarrow 0^{+}$, we get $x \in \overline{C_{1} \cap C_{2}}$.

(3) Let $x+y \in \overline{C_{1}}+\overline{C_{2}}$. Then there exist $x_{n} \in C_{1}$ and $y_{n} \in C_{2}$ such that $\lim _{n} x_{n}=x$ and $\lim _{n} y_{n}=y$. Then $(x+y)=\lim _{n}\left(x_{n}+y_{n}\right) \in \overline{C_{1}+C_{2}}$, which implies $\overline{C_{1}}+\overline{C_{2}} \subset \overline{C_{1}+C_{2}}$. Therefore,

$$
\overline{\overline{C_{1}}+\overline{C_{2}}} \subset \overline{\left(\overline{C_{1}+C_{2}}\right)}=\overline{C_{1}+C_{2}} \subset \overline{\overline{C_{1}}+\overline{C_{2}}}
$$

This proves (3) and we complete the proof of Lemma 2.3.

The following lemma shows that if the intersection of the $\varepsilon$-ball neighborhoods of convex sets is contained in the closure of a convex set $D$, then the intersection of smaller neighborhoods of these convex sets is contained in $D$. This lemma will be used to prove that certain properties of $\left\{\overline{C_{1}}, \ldots, \overline{C_{m}}\right\}$ also hold for $\left\{C_{1}, \ldots, C_{m}\right\}$.

Lemma 2.4. Let $C_{1}, \ldots, C_{m}, D$ be convex subsets of $H$ with $\bigcap_{1}^{m} C_{i} \neq \emptyset$. If

$$
\bigcap_{i=1}^{m}\left(C_{i}+\varepsilon B_{H}\right) \subset \bar{D}
$$

for some $\varepsilon>0$, then

$$
\bigcap_{i=1}^{m}\left(C_{i}+\hat{\varepsilon} B_{H}\right) \subset D \text { for all } 0<\hat{\varepsilon}<\varepsilon .
$$

Proof. It follows from 2.2 that

$$
\operatorname{int}\left[\bigcap_{i=1}^{m}\left(C_{i}+\varepsilon B_{H}\right)\right] \subset \operatorname{int}(\bar{D})=\operatorname{int}(D),
$$


where the equality follows from the fact that $\operatorname{int}(D)=\operatorname{int}(\bar{D})$ for any convex subset of $H$. Let $O_{H}$ be the open unit ball of $H$. Then

$$
\bigcap_{i=1}^{m}\left(C_{i}+\varepsilon O_{H}\right)=\bigcap_{i=1}^{m}\left(\bigcup_{x_{i} \in C_{i}}\left(x_{i}+\varepsilon O_{H}\right)\right)
$$

is an open set (since the union of open sets is open and a finite intersection of open sets is open). Therefore,

$$
\bigcap_{i=1}^{m}\left(C_{i}+\varepsilon O_{H}\right)=\operatorname{int}\left[\bigcap_{i=1}^{m}\left(C_{i}+\varepsilon O_{H}\right)\right] \subset \operatorname{int}\left[\bigcap_{i=1}^{m}\left(C_{i}+\varepsilon B_{H}\right)\right] \subset \operatorname{int}(D),
$$

where the last inclusion is (2.4). Since $\hat{\varepsilon} B_{H} \subset \varepsilon O_{H}$ for $0<\hat{\varepsilon}<\varepsilon$, (2.3) follows from (2.5).

It is well known that for convex cones, the strong CHIP (see Definition 5.1) holds at zero if and only if the strong CHIP holds for every point in the intersection of these cones (cf. [9]). This fact is actually a consequence of the following lemma concerning a particular inclusion property for the conical hulls of convex cones (see the proof of Theorem [5.4).

Lemma 2.5. Let $K_{1}, \ldots, K_{m}$ be convex cones in $H$ and let $D_{1}, D_{2}$ be subsets of H. If

$$
\bigcap_{i=1}^{m}\left(K_{i}+D_{1}\right) \subset \bigcap_{i=1}^{m} K_{i}+D_{2}
$$

then

$$
\bigcap_{i=1}^{m}\left(\operatorname{con}\left(K_{i}-x\right)+D_{1}\right) \subset \bigcap_{i=1}^{m} \operatorname{con}\left(K_{i}-x\right)+D_{2} \text { for all } x \in \bigcap_{i=1}^{m} K_{i} .
$$

Proof. Let $K:=\bigcap_{i=1}^{m} K_{i}$. Multiplying both sides of (2.6) by a positive number $\theta$, we get

$$
\bigcap_{i=1}^{m}\left(K_{i}+\theta D_{1}\right) \subset K+\theta D_{2}
$$

Subtracting any $x \in K$ from both sides of (2.8) and dividing both sides of it by $\theta$, we obtain

$$
\bigcap_{i=1}^{m}\left(\frac{1}{\theta}\left(K_{i}-x\right)+D_{1}\right) \subset \frac{1}{\theta}(K-x)+D_{2} .
$$

Since $x \in K$, we conclude that all sets $\frac{1}{\theta}\left(K_{i}-x\right)+D_{1}$ and $\frac{1}{\theta}(K-x)+D_{2}$ are decreasing as $\theta$ increases. Using this fact, Lemma 2.2, and Lemma 2.1(10), we obtain

$$
\begin{gathered}
\bigcap_{i=1}^{m}\left[\operatorname{con}\left(K_{i}-x\right)+D_{1}\right]=\bigcap_{i=1}^{m} \bigcup_{\theta>0}\left(\frac{1}{\theta}\left(K_{i}-x\right)+D_{1}\right)=\bigcup_{\theta>0} \bigcap_{i=1}^{m}\left(\frac{1}{\theta}\left(K_{i}-x\right)+D_{1}\right) \\
\subset \bigcup_{\theta>0}\left[\frac{1}{\theta}(K-x)+D_{2}\right] \subset \operatorname{con}(K-x)+D_{2}=\bigcap_{i=1}^{m} \operatorname{con}\left(K_{i}-x\right)+D_{2} .
\end{gathered}
$$

This completes the proof of Lemma 2.5. 
Finally, we include the following well known characterization for the projection of a point $x$ in $H$ onto a closed convex set $C$.

Lemma 2.6. Let $C$ be a closed convex subset of $H, x \in H$, and $x^{*} \in C$. Then $d(x, C)=\left\|x-x^{*}\right\|$ if and only if $x-x^{*} \in\left(C-x^{*}\right)^{\ominus}$. Furthermore, if $0 \in C$ and $d(x, C)=\left\|x-x^{*}\right\|$, then $\left\|x^{*}\right\| \leq\|x\|$.

Proof. The first statement of Lemma 2.6 is well-known (see, e.g., 29, Theorem 2.2.2 in Chapter II] or [16, Theorem 4.3, p. 44]). The second statement follows immediately from the nonexpansive property of the metric projection onto a closed convex set (see, e.g., [16, Theorem 5.5(5), p. 73]). For easy reference, we give the following simple proof. Assume $d(x, C)=\left\|x-x^{*}\right\|$ and $0 \in C$. Then $x-x^{*} \in$ $\left(C-x^{*}\right)^{\ominus}$, i.e.,

$$
\left\langle x-x^{*}, y-x^{*}\right\rangle \leq 0 \text { for all } y \in C .
$$

By substituting 0 for $y$ in (2.10), we get $\left\|x^{*}\right\|^{2} \leq\left\langle x, x^{*}\right\rangle \leq\|x\|\left\|x^{*}\right\|$, which implies $\left\|x^{*}\right\| \leq\|x\|$.

\section{VARIOUS NORMAL PROPERTIES}

In this section we extend the definition of normal property introduced by Jameson for two closed convex cones to a finite collection of convex sets. Variations of the normal property, such as the uniform normal property and the weak normal property, are also introduced. Then we study the hierarchy structure among various normal properties. These normal properties will be used later to study the geometric version of the basic constraint qualification in convex optimization and the linear regularity property of convex sets. We begin with the following definition of various normal properties.

Definition 3.1. Let $C_{1}, \ldots, C_{m}$ be convex sets in $H$ that have a nonempty intersection.

(1) $\left\{C_{1}, \ldots, C_{m}\right\}$ is said to have the closed intersection property if

$$
\overline{\bigcap_{i=1}^{m} C_{i}}=\bigcap_{i=1}^{m} \overline{C_{i}}
$$

(2) $\left\{C_{1}, \ldots, C_{m}\right\}$ is said to have the normal property if there exist $\varepsilon>0$ such that

$$
\bigcap_{i=1}^{m}\left(C_{i}+\varepsilon B_{H}\right) \subset\left(\bigcap_{i=1}^{m} C_{i}\right)+B_{H} .
$$

(3) $\left\{C_{1}, \ldots, C_{m}\right\}$ is said to have the weak normal property if for every $y \in H$ there exists $\varepsilon(y)>0$ such that

$$
\bigcap_{i=1}^{m}\left(C_{i}+\varepsilon(y) B_{H}\right) \subset\left(\bigcap_{i=1}^{m} C_{i}\right)+\{y\}^{\circ} .
$$

(4) $\left\{C_{1}, \ldots, C_{m}\right\}$ is said to have the uniform normal property if there exists a positive constant $\varepsilon$ such that

$$
\bigcap_{i=1}^{m}\left(C_{i}+\eta \varepsilon B_{H}\right) \subset\left(\bigcap_{i=1}^{m} C_{i}\right)+\eta B_{H} \text { for every } \eta>0 .
$$


Remark 3.1. (a) In 1940, M. Krein [35, Chapter V, Section 3, Subsection 3.2, Lemma 1] proved the first result concerning the strong CHIP for a pair of opposite convex cones $K_{1}=K$ and $K_{2}=-K$ in a Banach space. When restricted to the Hilbert space $H$, his result yields that $K_{1}^{\circ}+K_{2}^{\circ}=H=\{0\}^{\circ}=\left(K_{1} \cap K_{2}\right)^{\circ}$ when $\left\{K_{1}, K_{2}\right\}=\{K,-K\}$ is normal and $K \cap(-K)=\{0\}$. To extend Krein's result for two arbitrary convex cones, Jameson defined the normal property for two convex cones. He also used the abbreviation "property $(\mathrm{N})$ " for the normal property. Jameson regarded the normal property as a generalization of the notion of a normal cone (see [35]), and this motivated its name.

(b) In (2), (3), and (4) of Definition 3.1, one could replace $B_{H}$ by the open unit ball $O_{H}=\{x \in H \mid\|x\|<1\}$ everywhere to get equivalent definitions of various normal properties. We leave the details to the interested reader.

(c) In essence, the normal property means that if each $C_{i}$ is expanded outward by an $\varepsilon$-ball, then the intersection of the expanded sets is within a fixed distance from the intersection of the original sets. It is interesting to note that the "reverse" version of the normal property was also studied in convex optimization, which is called the bounded excess property. Let $g_{i}(x)$ be convex functions on $H$ and define $C_{i}:=\left\{x \in H \mid g_{i}(x) \leq 0\right\}$. Then $\left\{C_{1}, \ldots, C_{m}\right\}$ is said to have the bounded excess property if there exist $\delta>0$ and $\varepsilon>0$ such that

$$
\bigcap_{i=1}^{m} C_{i} \subset\left(\bigcap_{i=1}^{m} C_{i}(-\varepsilon)\right)+\delta B_{H}
$$

where $C_{i}(-\varepsilon):=\left\{x \in H \mid g_{i}(x) \leq-\varepsilon\right\}$ can be considered as the algebraic version of shrinking $C_{i}$ inward by an $\varepsilon$-ball. It was proved that (3.5) implies the existence of a global error bound for approximate solutions of the convex feasibility problem: $g_{i}(x) \leq 0$ for $1 \leq i \leq m$. That is,

$$
d\left(x, \bigcap_{i=1}^{m} C_{i}\right) \leq \gamma \sum_{i=1}^{m} \max \left\{g_{i}(x), 0\right\} \text { for all } x \in H,
$$

where $\gamma>0$ is a positive constant. See [13, 14] and [25, Theorem 5] for details. It is not clear whether there is a relationship between the normal property and the geometric version of the bounded excess property of (3.5), where $C_{i}(-\varepsilon)$ is defined as the set obtained by shrinking $C_{i}$ inward by an $\varepsilon$-ball:

$$
C_{i}(-\varepsilon):=\left\{x \in C_{i} \mid d\left(x,\left(H \backslash C_{i}\right) \cap \operatorname{aff}\left(C_{i}\right)\right) \geq \varepsilon\right\} .
$$

Our conjecture is that the bounded excess property implies the uniform normal property, but the converse is not true. (After this paper had been submitted for publication, Hein Hundal privately communicated a counterexample to this conjecture.)

The following theorem shows a hierarchy of various normal properties for a finite collection of convex sets.

Theorem 3.1. Let $C_{1}, \ldots, C_{m}$ (respectively $K_{1}, \ldots, K_{m}$ ) be convex sets (respectively convex cones).

(1) If each $C_{i}$ is closed, then $\left\{C_{1}, \ldots, C_{m}\right\}$ has the closed intersection property.

(2) If $\left\{C_{1}, \ldots, C_{m}\right\}$ has the uniform normal property, then it has the normal property and the closed intersection property. 
(3) $\left\{K_{1}, \ldots, K_{m}\right\}$ has the normal property if and only if it has the uniform normal property.

(4) If $\left\{K_{1}, \ldots, K_{m}\right\}$ has the normal property (or, equivalently, the uniform normal property), then it has the weak normal property and the closed intersection property.

(5) $\left\{K_{1}, \ldots, K_{m}\right\}$ has the normal property, the weak normal property, or the uniform normal property if and only if it has the closed intersection property and $\left\{\bar{K}_{1}, \ldots, \bar{K}_{m}\right\}$ has the normal property, the weak normal property, or the uniform normal property, respectively.

Proof. Statement (1) is trivial. To verify (2), let $\left\{C_{1}, \ldots, C_{m}\right\}$ have the uniform normal property. Then clearly it has the normal property (take $\eta=1$ ). If we intersect both sides of equation (3.4) over all $\eta>0$, we obtain that $\bigcap_{1}^{m} \overline{C_{i}} \subset \overline{\bigcap_{1}^{m} C_{i}}$. Since the reverse inclusion is obvious, it follows that $\left\{C_{1}, \ldots, C_{m}\right\}$ has the closed intersection property, and this verifies statement (2).

If $\left\{K_{1}, \ldots, K_{m}\right\}$ has the normal property, multiply both sides of relation (3.2) (with $C_{i}=K_{i}$ ) by $\eta>0$ to obtain the relation (3.4) (with $C_{i}=K_{i}$ ), and this shows that $\left\{K_{1}, \ldots, K_{m}\right\}$ has the uniform normal property. Since the uniform normal property implies the normal property by (2), it follows that (3) holds.

To prove (4), suppose that $\left\{K_{1}, \ldots, K_{m}\right\}$ has the normal property, and let $y \in H$. If $\|y\| \leq 1$, then $B_{H} \subset\{y\}^{\circ}$, so that relation (3.3) holds with $\varepsilon(y)=\varepsilon$. If $\|y\|>1$, then multiplying both sides of relation (3.2) by $1 /\|y\|$, we obtain that

$$
\bigcap_{i=1}^{m}\left(K_{i}+\frac{\varepsilon}{\|y\|} B_{H}\right) \subset \bigcap_{i=1}^{m} K_{i}+\frac{1}{\|y\|} B_{H} .
$$

But since $\frac{1}{\|y\|} B_{H} \subset\{y\}^{\circ}$, it follows that (3.3) holds with $\varepsilon(y)=\varepsilon /\|y\|$. Thus, $\left\{K_{1}, \ldots, K_{m}\right\}$ has the weak normal property, and (4) is verified.

If $\left\{K_{1}, \ldots, K_{m}\right\}$ has the normal property, then it has the uniform normal property by (3), and hence the closed intersection property by (2). Using the normal property, we have that

$$
\bigcap_{i=1}^{m}\left(\bar{K}_{i}+\frac{\varepsilon}{2} B_{H}\right) \subset \bigcap_{i=1}^{m}\left(K_{i}+\varepsilon B_{H}\right) \subset \bigcap_{i=1}^{m} K_{i}+B_{H} \subset \bigcap_{i=1}^{m} \bar{K}_{i}+B_{H} .
$$

This proves that $\left\{\bar{K}_{1}, \ldots, \bar{K}_{m}\right\}$ has the normal property (with $\varepsilon / 2$ instead of $\varepsilon$ ).

Conversely, assume that $\left\{K_{1}, \ldots, K_{m}\right\}$ has the closed intersection property and that $\left\{\bar{K}_{1}, \ldots, \bar{K}_{m}\right\}$ has the normal property. Then there exists $\varepsilon>0$ such that

$$
\bigcap_{i=1}^{m}\left(\bar{K}_{i}+\varepsilon B_{H}\right) \subset \bigcap_{i=1}^{m} \bar{K}_{i}+B_{H}
$$

Now we can derive that

$$
\bigcap_{i=1}^{m}\left(K_{i}+\varepsilon B_{H}\right) \subset \bigcap_{i=1}^{m}\left(\bar{K}_{i}+\varepsilon B_{H}\right) \subset \bigcap_{i=1}^{m} \bar{K}_{i}+B_{H}=\overline{\bigcap_{i=1}^{m} K_{i}}+B_{H} \subset \overline{\bigcap_{i=1}^{m} K_{i}+B_{H}},
$$

where the first inclusion is obviously true, the second inclusion is (3.7), the equality is by the closed intersection property, and the last inclusion follows from Lemma 
2.3(3). Applying Lemma 2.4 with $C_{i}=K_{i}$ and $D=\left(\bigcap_{i=1}^{m} K_{i}\right)+B_{H}$, we get

$$
\bigcap_{i=1}^{m}\left(K_{i}+\hat{\varepsilon} B_{H}\right) \subset \bigcap_{i=1}^{m} K_{i}+B_{H} \text { for all } 0<\hat{\varepsilon}<\varepsilon,
$$

which implies that $\left\{K_{1}, \ldots, K_{m}\right\}$ has the normal property. Thus, the statement in (5) concerning the normal property is verified. Using (4), it follows that the statement in (5) concerning the uniform normal property is also valid.

It remains to verify the statement in (5) concerning the weak normal property. First assume that $\left\{K_{1}, \ldots, K_{m}\right\}$ has the weak normal property. Then for each $y \in H$, there exists $\varepsilon(y)>0$ such that

$$
\bigcap_{i=1}^{m}\left(\bar{K}_{i}+\frac{\varepsilon(y)}{2} B_{H}\right) \subset \bigcap_{i=1}^{m}\left(K_{i}+\varepsilon(y) B_{H}\right) \subset \bigcap_{i=1}^{m} K_{i}+\{y\}^{\circ} \subset \bigcap_{i=1}^{m} \bar{K}_{i}+\{y\}^{\circ} .
$$

This proves that $\left\{\bar{K}_{1}, \ldots, \bar{K}_{m}\right\}$ has the weak normal property, where $\bar{\varepsilon}(y):=\varepsilon(y) / 2$ works for each $y \in H$.

To show that $\left\{K_{1}, \ldots, K_{m}\right\}$ has the closed intersection property, we intersect both sides of the inclusion in (3.3) over all $y \in H$ to get

$$
\bigcap_{i=1}^{m} \bar{K}_{i} \subset \bigcap_{y \in H} \bigcap_{i=1}^{m}\left(K_{i}+\varepsilon(y) B_{H}\right) \subset \bigcap_{y \in H}\left(\bigcap_{i=1}^{m} K_{i}+\{y\}^{\circ}\right) .
$$

Next we prove that the last set in (3.9) is contained in $\bar{K}:=\overline{\bigcap_{1}^{m} K_{i}}$. Let $z \notin \bar{K}$. Since $d(z, \bar{K})>0$, for any $0<\rho<d(z, \bar{K})$, we have $\left(z+\rho B_{H}\right) \cap \bar{K}=\emptyset$. By the first separation theorem (see [35, Ch.II, §9] or [16, Theorem 6.23, p. 103]) applied to $\bar{K}$ and $z+\rho B_{H}$, one can find $\bar{y} \in H$ such that $\langle\bar{y}, \bar{K}\rangle \leq 0<1<\langle\bar{y}, z\rangle$, which implies that $\langle\bar{y}, z-x\rangle>1$ for $x \in \bar{K}$. Thus, $z \notin \bar{K}+\{\bar{y}\}^{\circ}$. This proves that

$$
\bigcap_{y \in H}\left[\bigcap_{i=1}^{m} K_{i}+\{y\}^{\circ}\right] \subset \bar{K}=\overline{\bigcap_{i=1}^{m} K_{i}}
$$

It follows from (3.9) and (3.10) that $\bigcap_{i=1}^{m} \bar{K}_{i} \subset \overline{\bigcap_{i=1}^{m} K_{i}}$. But $\overline{\bigcap_{i=1}^{m} K_{i}} \subset \bigcap_{i=1}^{m} \bar{K}_{i}$. So $\bigcap_{1}^{m} \bar{K}_{i}=\overline{\bigcap_{1}^{m} K_{i}}$ and $\left\{K_{1}, \ldots, K_{m}\right\}$ has the closed intersection property.

Finally, assume that $\left\{\bar{K}_{1}, \ldots, \bar{K}_{m}\right\}$ has the weak normal property and that $\left\{K_{1}, \ldots, K_{m}\right\}$ has the closed intersection property. We must show that $\left\{K_{1}, \ldots\right.$, $\left.K_{m}\right\}$ has the weak normal property. For each $y \in H$, there exists $\bar{\varepsilon}(y)>0$ such that

$$
\bigcap_{i=1}^{m}\left(\bar{K}_{i}+\bar{\varepsilon}(y) B_{H}\right) \subset \bigcap_{i=1}^{m} \bar{K}_{i}+\{y\}^{\circ} .
$$

Then

$$
\begin{aligned}
\bigcap_{i=1}^{m}\left(K_{i}+\bar{\varepsilon}(y) B_{H}\right) & \subset \bigcap_{i=1}^{m}\left(\bar{K}_{i}+\bar{\varepsilon}(y) B_{H}\right) \subset\left[\bigcap_{i=1}^{m} \bar{K}_{i}+\{y\}^{\circ}\right] \\
& =\bigcap_{i=1}^{m} K_{i}+\{y\}^{\circ} \subset \bigcap_{i=1}^{m} K_{i}+\{y\}^{\circ}
\end{aligned}
$$

where the first inclusion is obviously true, the second inclusion is (3.11), the equality is by the closed intersection property, and the last inclusion follows from Lemma 
2.3(3). Applying Lemma 2.4 with $C_{i}=K_{i}, \varepsilon=\bar{\varepsilon}(y)$, and $D=\left(\bigcap_{i=1}^{m} K_{i}\right)+\{y\}^{\circ}$, we get

$$
\bigcap_{i=1}^{m}\left(K_{i}+\bar{\varepsilon} B_{H}\right) \subset \bigcap_{i=1}^{m} K_{i}+\{y\}^{\circ} \text { for all } 0<\bar{\varepsilon}<\bar{\varepsilon}(y),
$$

which implies that $\left\{K_{1}, \ldots, K_{m}\right\}$ has the weak normal property. This completes the proof of Theorem 3.1 .

\section{Dual normality}

In this section, we study a dual form of the normal property, which is a generalization of the property $(\mathrm{G})$ that was introduced by Jameson for closed convex cones. A quantitative relationship between the normal property and the dual normal property is established in Theorem 4.1. To explore the quantitative relationship explicitly, we define the normality constant and the dual normality constant for a finite collection of convex sets $\left\{C_{1}, \ldots, C_{m}\right\}$ in Definition 4.2. If $\left\{C_{1}, \ldots, C_{m}\right\}$ has the closed intersection property, then the normality constant for $\left\{C_{1}, \ldots, C_{m}\right\}$ is the same as the dual normality constant for its collection of polars $\left\{C_{1}^{\circ}, \ldots, C_{m}^{\circ}\right\}$ (see Theorem 4.2). In the special case when all the sets are closed convex cones, the dual normal property is actually equivalent to property $(\mathrm{G})$ introduced by Jameson (see Theorem 4.3 and Corollary 4.1). In the next section, we will see how the dual normal property can be used to characterize the linear regularity of $\left\{C_{1}, \ldots, C_{m}\right\}$.

The main technical difficulty in this section is to prove a few facts about the polar of the sum of two convex sets. This turns out to involve the "inverse sum" of two convex sets. Therefore, let us define the inverse sum of two convex sets and study some of its properties.

Definition 4.1. Let $D_{1}$ and $D_{2}$ be convex subsets of $H$ with $0 \in D_{1} \cap D_{2}$. Then the inverse sum of $D_{1}$ and $D_{2}$ is defined as the union of the convex sets [ $\left.t D_{1} \cap(1-t) D_{2}\right]$ $(0<t<1)$ and their limits (with respect to $t$ as $t \rightarrow 0^{+}$and $t \rightarrow 1^{-}$); that is,

$$
D_{1} \# D_{2}:=\left(\bigcup_{0<t<1}\left[t D_{1} \cap(1-t) D_{2}\right]\right) \cup\left(D_{1} \cap 0^{+} D_{2}\right) \cup\left(D_{2} \cap 0^{+} D_{1}\right) .
$$

Remark 4.1. The definition and notation for the inverse sum are adopted from the book of Kusraev and Kutateladze [28, §1.1.6(8)]. One can find a detailed exposition of the inverse sum and its applications in this book. We should note that Rockafellar [34, section 3 of Chapter 1] defined the inverse sum somewhat differently than this. He essentially dropped the two terms involving the recession directions, i.e., his inverse sum was defined to be the set of all proper convex combinations of elements of $D_{1}$ and $D_{2}$. Of course, in case both $D_{1}$ and $D_{2}$ are bounded, then $0^{+} D_{i}=\{0\}$ for $i=1,2$ and the two definitions agree.

In the following lemma we list some facts about inverse sums of convex sets, which will be used to derive a dual form of the normal property.

Lemma 4.1. Let $C_{1}$ and $C_{2}$ be convex subsets of $H$ with $0 \in C_{1} \cap C_{2}$.

(1) $C_{1} \# C_{2}$ is a convex set.

(2) $C_{1} \# C_{2} \subset C_{1} \cap C_{2}$.

(3) If $C_{1}$ is a cone, then $C_{1} \# C_{2}=C_{1} \cap C_{2}$.

(4) If $C_{1}$ and $C_{2}$ are closed, then $C_{1} \# C_{2}$ is closed.

(5) If $C_{1}$ is closed and bounded with $0 \in \operatorname{int}\left(C_{1}\right)$, then $C_{1} \# \overline{C_{2}} \subset \overline{C_{1} \# C_{2}}$. 
(6) If $C_{1}$ and $C_{2}$ are closed and $C_{1} \cap C_{2}$ is bounded, then $C_{1} \# C_{2}$ is a weakly compact set.

(7) $\left(C_{1}+C_{2}\right)^{\circ}=C_{1}^{\circ} \# C_{2}^{\circ}$.

Proof. (1) This follows from the definition (see [28, §1.1.7]).

(2) Since $0 \in C_{1} \cap C_{2}$, for any $0<t<1$, we have $t C_{1} \subset C_{1}$, $(1-t) C_{2} \subset C_{2}$, $0^{+} C_{1} \subset C_{1}$, and $0^{+} C_{2} \subset C_{2}$. Thus, $C_{1} \# C_{2} \subset C_{1} \cap C_{2}$.

(3) Since $C_{1}$ is a cone, $0^{+} C_{1}=C_{1}$. Thus, $C_{1} \cap C_{2}=0^{+} C_{1} \cap C_{2} \subset C_{1} \# C_{2}$. By (2), we have $C_{1} \cap C_{2}=C_{1} \# C_{2}$.

(4) Let $\left\{x_{n}\right\} \subset C_{1} \# C_{2}$ converge to $x^{*}$. We must prove that $x^{*} \in C_{1} \# C_{2}$. Since $C_{1}$ and $C_{2}$ are closed convex sets in $H, C_{1} \cap 0^{+} C_{2}$ is closed. Therefore, if there exist infinitely many indices $n$ such that $x_{n} \in C_{1} \cap 0^{+} C_{2}$, then $x^{*} \in C_{1} \cap 0^{+} C_{2} \subset$ $C_{1} \# C_{2}$. Similarly, if there exist infinitely many indices $n$ such that $x_{n} \in C_{2} \cap 0^{+} C_{1}$, then $x^{*} \in C_{2} \cap 0^{+} C_{1} \subset C_{1} \# C_{2}$. Otherwise, by deleting those $x_{n}$ 's that are in $\left(C_{1} \cap 0^{+} C_{2}\right) \cup\left(C_{2} \cap 0^{+} C_{1}\right)$ from the sequence, we may assume that there exists a sequence $\left\{t_{n}\right\}$ such that $0<t_{n}<1$ and $x_{n} \in t_{n} C_{1} \cap\left(1-t_{n}\right) C_{2}$. Again, by replacing $\left\{t_{n}\right\}$ with a convergent subsequence, we may assume that $\lim _{n \rightarrow \infty} t_{n}=t \in[0,1]$.

First assume $t=0$. Let $\gamma>0$ be any fixed positive number. Since $t_{n} \rightarrow 0$, for $n$ sufficiently large we have $\gamma<1 / t_{n}$, which implies $\gamma x_{n} \in \gamma t_{n} C_{1} \subset C_{1}$ (since $0 \in C_{1}$ ). By taking the limit as $n \rightarrow \infty$, we obtain $\gamma x^{*} \in C_{1}$ (by the closedness of $C_{1}$ ). Since $\gamma>0$ is arbitrary, we get $x^{*} \in 0^{+} C_{1}$. Since $x_{n} \in\left(1-t_{n}\right) C_{2} \subset C_{2}$ (since $0 \in C_{2}$ ) and $C_{2}$ is closed, we obtain $x^{*} \in C_{2}$. It follows that $x^{*} \in C_{2} \cap 0^{+} C_{1} \subset C_{1} \# C_{2}$. Similarly, if $t=1$, then $x^{*} \in C_{1} \cap 0^{+} C_{2} \subset C_{1} \# C_{2}$.

If $0<t<1$, the closedness of $C_{1}$ and $C_{2}$ implies that $x_{n} / t_{n} \in C_{1}$ converges to $x^{*} / t \in C_{1}$ and $x_{n} /\left(1-t_{n}\right) \in C_{2}$ converges to $x^{*} /(1-t) \in C_{2}$. Thus, $x^{*} \in$ $t C_{1} \cap(1-t) C_{2} \subset C_{1} \# C_{2}$. This proves that $x^{*} \in C_{1} \# C_{2}$. Hence, $C_{1} \# C_{2}$ is closed.

(5) Since $0 \in \operatorname{int}\left(C_{1}\right)$, we have $0 \in \operatorname{int}\left(t C_{1}\right)$ for any $t>0$. Let $0<t<1$ and $x \in t C_{1} \cap(1-t) \overline{C_{2}}$. Then

$$
t C_{1} \cap(1-t) \overline{C_{2}}=\overline{t C_{1}} \cap \overline{(1-t) C_{2}}=\overline{t C_{1} \cap(1-t) C_{2}} \subset \overline{C_{1} \# C_{2}} \text { for all } 0<t<1,
$$

where the second equality follows from $0 \in(1-t) C_{2} \cap \operatorname{int}\left(t C_{1}\right)$ and Lemma 2.3(2), while the last inclusion follows from the definition of $C_{1} \# C_{2}$. Similarly, we have

$$
C_{1} \cap 0^{+} \overline{C_{2}}=C_{1} \cap \overline{0^{+} C_{2}}=\overline{C_{1} \cap 0^{+} C_{2}} \subset \overline{C_{1} \# C_{2}},
$$

where the first equality follows from the definition of the recession cone and the second equality follows from $0 \in 0^{+} C_{2} \cap \operatorname{int}\left(C_{1}\right)$ and Lemma 2.3](2). Since $C_{1}$ is a bounded set, we have $0^{+} C_{1}=\{0\}$, so $\overline{C_{2}} \cap 0^{+} C_{1}=\{0\} \subset \overline{C_{1} \# C_{2}}$. This proves that $C_{1} \# \overline{C_{2}} \subset \overline{C_{1} \# C_{2}}$.

(6) It follows from (1), (2), and (4) that $C_{1} \# C_{2}$ is a closed bounded convex set in $H$, so it is weakly compact (see [16, Theorems 9.12 and 9.16]).

(7) This is the formula (3) in [28, §3.3.12]. However, the proof given in [28] is quite complicated, involving infimal convolution and subdifferential formulas for convex functions. Therefore, we give the following elementary proof of (7).

Let $0<t<1$ and $x \in t C_{1}^{\circ} \cap(1-t) C_{2}^{\circ}$. Then $\left\langle x, C_{1}+C_{2}\right\rangle=\left\langle x, C_{1}\right\rangle+\left\langle x, C_{2}\right\rangle \leq$ $t+(1-t)=1$, i.e., $x \in\left(C_{1}+C_{2}\right)^{\circ}$. Thus, $\bigcup_{0<t<1}\left[t C_{1}^{\circ} \cap(1-t) C_{2}^{\circ}\right] \subset\left(C_{1}+C_{2}\right)^{\circ}$. If $x \in C_{2}^{\circ} \cap 0^{+} C_{1}^{\circ}=C_{2}^{\circ} \cap C_{1}^{\ominus}$, then $\left\langle x, C_{1}+C_{2}\right\rangle=\left\langle x, C_{1}\right\rangle+\left\langle x, C_{2}\right\rangle \leq 0+1=1$, so $C_{2}^{\circ} \cap 0^{+} C_{1}^{\circ} \subset\left(C_{1}+C_{2}\right)^{\circ}$. Similarly, $C_{1}^{\circ} \cap 0^{+} C_{2}^{\circ} \subset\left(C_{1}+C_{2}\right)^{\circ}$. This proves $C_{1}^{\circ} \# C_{2}^{\circ} \subset\left(C_{1}+C_{2}\right)^{\circ}$. 
Conversely, if $x \in\left(C_{1}+C_{2}\right)^{\circ}$, then

$$
\sup _{y_{1} \in C_{1}}\left\langle x, y_{1}\right\rangle+\sup _{y_{2} \in C_{2}}\left\langle x, y_{2}\right\rangle=\sup _{y_{1} \in C_{1}, y_{2} \in C_{2}}\left\langle x, y_{1}+y_{2}\right\rangle=\sup _{y \in C_{1}+C_{2}}\langle x, y\rangle \leq 1 .
$$

Let $t=\sup _{y_{1} \in C_{1}}\left\langle x, y_{1}\right\rangle$. Since $0 \in C_{1} \cap C_{2}$, we have $\sup _{y_{i} \in C_{i}}\left\langle x, y_{i}\right\rangle \geq 0$. Therefore, (4.2) implies $0 \leq t \leq 1$. If $t=0, x \in C_{1}^{\ominus}$. Moreover, for $t=0$, (4.2) implies $x \in C_{2}^{\circ}$, so $x \in C_{2}^{\circ} \cap C_{1}^{\ominus}=C_{2}^{\circ} \cap 0^{+} C_{1}^{\circ} \subset C_{1}^{\circ} \# C_{2}^{\circ}$. Similarly, if $t=1$, then $x \in C_{1}^{\circ} \cap C_{2}^{\ominus}=C_{1}^{\circ} \cap 0^{+} C_{2}^{\circ} \subset C_{1}^{\circ} \# C_{2}^{\circ}$. Finally, If $0<t<1$, then (4.2) implies $\sup _{y_{2} \in C_{2}}\left\langle x, y_{2}\right\rangle \leq 1-t$. Since $t>0$ and $1-t>0$, we have $x \in t C_{1}^{\circ} \cap(1-t) C_{2}^{\circ} \subset$ $C_{1}^{\circ} \# C_{2}^{\circ}$. This completes the proof of (7).

Remark 4.2. If $D_{1}$ and $D_{2}$ are closed convex cones, then it follows from statements (1) and (4) of Lemma 4.1 that

$$
D_{1} \# D_{2}=\overline{\operatorname{co}}\left(\bigcup_{0<t<1}\left[t D_{1} \cap(1-t) D_{2}\right]\right)=\overline{\left(\bigcup_{0<t<1}\left[t D_{1} \cap(1-t) D_{2}\right]\right)} .
$$

Actually, one can prove the following formula: if $D_{1}$ and $D_{2}$ are convex cones, then

$$
\overline{D_{1} \# D_{2}}=\overline{\operatorname{co}}\left(\bigcup_{0<t<1}\left[t D_{1} \cap(1-t) D_{2}\right]\right)=\overline{\left(\bigcup_{0<t<1}\left[t D_{1} \cap(1-t) D_{2}\right]\right)} .
$$

We leave the details to the interested reader. However, in general, $\overline{D_{1} \# D_{2}} \neq$ $\overline{D_{1}} \# \overline{D_{2}}$. For example, consider the following two convex cones in $\mathbb{R}^{2}$ :

$D_{1}=\left\{(t, 0) \in \mathbb{R}^{2} \mid t \in \mathbb{R}\right\} \quad$ and $\quad D_{2}=\left\{\left(t_{1}, t_{2}\right) \in \mathbb{R}^{2} \mid t_{1}, t_{2} \in \mathbb{R}, t_{2}>0\right\} \cup\{(0,0)\}$.

Note that $D_{2}$ is not closed. It is easy to verify that

$$
D_{1} \# D_{2}=\overline{D_{1} \# D_{2}}=\{(0,0)\} \neq D_{1}=\overline{D_{1}} \cap \overline{D_{2}}=\overline{D_{1}} \# \overline{D_{2}} .
$$

One major step in establishing the dual form of the normal property is to compute the polar of $\bigcap_{i=1}^{m}\left(C_{i}+\varepsilon B_{H}\right)$ for convex sets $C_{i}$. This will also be used later to derive the dual form of the weak normal property. For ease of reference, we now give an expression for the polar of $\bigcap_{i=1}^{m}\left(C_{i}+\varepsilon B_{H}\right)$ in terms of the polars of the $C_{i}$ 's.

Lemma 4.2. Let $C_{1}, \ldots, C_{m}$ be convex sets with $0 \in \bigcap_{i=1}^{m} C_{i}$. Then

$$
\left(\bigcap_{i=1}^{m}\left[C_{i}+\varepsilon B_{H}\right]\right)^{\circ}=\operatorname{co}\left(\bigcup_{i=1}^{m}\left(C_{i}^{\circ} \# \frac{1}{\varepsilon} B_{H}\right)\right) .
$$

Proof. We have that

$$
\begin{aligned}
& \left(\bigcap_{i=1}^{m}\left[C_{i}+\varepsilon B_{H}\right]\right)^{\circ}=\left(\overline{\bigcap_{i=1}^{m}\left[C_{i}+\varepsilon B_{H}\right]}\right)^{\circ} \\
& \quad=\left(\bigcap_{i=1}^{m} \overline{\left[C_{i}+\varepsilon B_{H}\right]}\right)^{\circ}=\overline{\mathrm{co}}\left[\bigcup_{i=1}^{m}\left(\overline{C_{i}+\varepsilon B_{H}}\right)^{\circ}\right] \\
& \quad=\overline{\mathrm{co}}\left[\bigcup_{i=1}^{m}\left(C_{i}+\varepsilon B_{H}\right)^{\circ}\right]=\overline{\mathrm{co}}\left[\bigcup_{i=1}^{m}\left(C_{i}^{\circ} \# \frac{1}{\varepsilon} B_{H}\right)\right]=\operatorname{co}\left[\bigcup_{i=1}^{m}\left(C_{i}^{\circ} \# \frac{1}{\varepsilon} B_{H}\right)\right],
\end{aligned}
$$

where the first and fourth equalities are by Lemma 2.1(1), the second equality is by Lemma 2.3(2), the third equality is by Lemma 2.17), and the fifth equality is 
by Lemma 4.1(7). By Lemma $4.1(6)$, each set $C_{i}^{\circ} \# \frac{1}{\varepsilon} B_{H}$ is weakly compact. Since a finite union of weakly compact sets is also weakly compact, the last equality in (4.7) follows from Lemma 2.1(11). This completes the proof of Lemma 4.2.

Now we can formulate the dual form of the normal property for a finite collection of convex sets. The equivalence of the normal property and its dual form is not exact. The main effort is to derive the dual form without the closure operation involved, which creates some technical difficulty in the proof.

Theorem 4.1. Let $C_{1}, \ldots, C_{m}$ be convex subsets of $H$ and let $0 \in \bigcap_{i=1}^{m} C_{i}$. Assume that $\varepsilon$ is a positive constant. If

$$
\bigcap_{i=1}^{m}\left(C_{i}+\varepsilon B_{H}\right) \subset\left(\bigcap_{i=1}^{m} C_{i}\right)+B_{H}
$$

then

$$
B_{H} \# \operatorname{co}\left(\bigcup_{i=1}^{m} C_{i}^{\circ}\right) \subset \operatorname{co}\left(\bigcup_{i=1}^{m}\left(C_{i}^{\circ} \# \frac{1}{\varepsilon} B_{H}\right)\right) .
$$

Conversely, if (4.9) holds and $\left\{C_{1}, \ldots, C_{m}\right\}$ has the closed intersection property, then

$$
\bigcap_{i=1}^{m}\left(C_{i}+\hat{\varepsilon} B_{H}\right) \subset\left(\bigcap_{i=1}^{m} C_{i}\right)+B_{H} \text { for all } 0<\hat{\varepsilon}<\varepsilon .
$$

Proof. Applying the polar operation to both sides of (4.8) and using Lemma 2.17) together with Lemma 4.1(7), we obtain

$$
B_{H} \# \overline{\mathrm{co}}\left(\bigcup_{i=1}^{m} C_{i}^{\circ}\right) \subset\left[\bigcap_{i=1}^{m}\left(C_{i}+\varepsilon B_{H}\right)\right]^{\circ} .
$$

By Lemma 4.2 (4.11) can be rewritten as follows:

$$
B_{H} \# \overline{\mathrm{co}}\left(\bigcup_{i=1}^{m} C_{i}^{\circ}\right) \subset \operatorname{co}\left(\bigcup_{i=1}^{m}\left(C_{i}^{\circ} \# \frac{1}{\varepsilon} B_{H}\right)\right),
$$

which obviously implies 4.9).

Conversely, if (4.9) holds, by applying the polar operation to both sides of (4.9) and using Lemma 4.2, we obtain

$$
\left[\bigcap_{i=1}^{m}\left(C_{i}+\varepsilon B_{H}\right)\right]^{\circ} \subset\left[B_{H} \# \operatorname{co}\left(\bigcup_{i=1}^{m} C_{i}^{\circ}\right)\right]^{\circ} .
$$

But

$$
\begin{gathered}
{\left[B_{H} \# \mathrm{co}\left(\bigcup_{i=1}^{m} C_{i}^{\circ}\right)\right]^{\circ}=\left[\overline{B_{H} \# \operatorname{co}\left(\bigcup_{i=1}^{m} C_{i}^{\circ}\right)}\right]^{\circ} \subset\left[B_{H} \# \overline{\mathrm{co}}\left(\bigcup_{i=1}^{m} C_{i}^{\circ}\right)\right]^{\circ}} \\
=\left[B_{H}+\bigcap_{i=1}^{m} C_{i}^{\circ \circ}\right]^{\circ}=\overline{B_{H}+\bigcap_{i=1}^{m} \overline{C_{i}}}=\overline{B_{H}+\bigcap_{i=1}^{m} C_{i}}=\overline{B_{H}+\bigcap_{i=1}^{m} C_{i}}
\end{gathered}
$$

where the first equality is by Lemma $[2.1$ (1), the inclusion follows from Lemma 4.1(5), the second equality follows from Lemma 4.1(7) and Lemma 2.17), the third 
equality is by Lemma 2.1 4 ), the closed intersection property of $\left\{C_{1}, \ldots, C_{m}\right\}$ yields the fourth equality, and the last equality is by Lemma[2.3](3). It follows from Lemma 2.1(4), (4.13), and (4.14) that

$$
\bigcap_{i=1}^{m}\left(C_{i}+\varepsilon B_{H}\right) \subset\left[\bigcap_{i=1}^{m}\left(C_{i}+\varepsilon B_{H}\right)\right]^{\circ} \subset \overline{\left(\bigcap_{i=1}^{m} C_{i}\right)+B_{H}} .
$$

Applying Lemma 2.4 with $D=\bigcap_{i=1}^{m} C_{i}+B_{H}$, we get (4.10). This completes the proof of Theorem 4.1

The above theorem does not give a complete quantitative characterization of the normal property in dual form. To get a better understanding of the duality relationship for the normal property, we introduce the following quantitative measurement of the normality for a finite collection of convex sets.

Definition 4.2. The normality constant for a finite collection of convex sets $\left\{C_{1}, \ldots, C_{m}\right\}$ in $H$ is defined as follows:

$$
\lambda_{N}\left(C_{1}, \ldots, C_{m}\right):=\sup \left\{\varepsilon \geq 0 \mid \bigcap_{i=1}^{m}\left(C_{i}+\varepsilon B_{H}\right) \subset\left(\bigcap_{i=1}^{m} C_{i}\right)+B_{H}\right\} .
$$

The dual normality constant for a finite collection of convex sets $\left\{C_{1}, \ldots, C_{m}\right\}$ in $H$ is defined by

$$
\lambda_{D}\left(C_{1}, \ldots, C_{m}\right):=\sup \left\{\varepsilon \geq 0 \mid B_{H} \# \text { co }\left(\bigcup_{i=1}^{m} C_{i}\right) \subset \operatorname{co}\left(\bigcup_{i=1}^{m}\left(C_{i} \# \frac{1}{\varepsilon} B_{H}\right)\right)\right\},
$$

where $\frac{1}{\varepsilon} B_{H}$ is defined to be the whole space $H$ when $\varepsilon=0$. We will say that $\left\{C_{1}, \ldots, C_{m}\right\}$ has the dual normal property if $\lambda_{D}\left(C_{1}, \ldots, C_{m}\right)>0$.

Obviously, $\left\{C_{1}, \ldots, C_{m}\right\}$ has the normal property if and only if $\lambda_{N}\left(C_{1}, \ldots, C_{m}\right)$ $>0$. The following reformulation of Theorem 4.1 in terms of normality constants shows an interesting dual relationship that is hidden in Theorem 4.1.

Theorem 4.2. Let $\left\{C_{1}, \ldots, C_{m}\right\}$ be a collection of convex sets with the closed intersection property and let $0 \in \bigcap_{1}^{m} C_{i}$. Then

$$
\lambda_{N}\left(C_{1}, \ldots, C_{m}\right)=\lambda_{D}\left(C_{1}^{\circ}, \ldots, C_{m}^{\circ}\right) .
$$

Proof. By Theorem 4.1, the implication (4.8) $\Rightarrow(4.9)$ shows that

$$
0 \leq \lambda_{N}\left(C_{1}, \ldots, C_{m}\right) \leq \lambda_{D}\left(C_{1}^{\circ}, \ldots, C_{m}^{\circ}\right) .
$$

If $\lambda_{D}\left(C_{1}^{\circ}, \ldots, C_{m}^{\circ}\right)=0$, then (4.16) holds. Otherwise, we have

$$
\lambda_{D}\left(C_{1}^{\circ}, \ldots, C_{m}^{\circ}\right)-\delta \leq \lambda_{N}\left(C_{1}, \ldots, C_{m}\right) \leq \lambda_{D}\left(C_{1}^{\circ}, \ldots, C_{m}^{\circ}\right),
$$

for any $0<\delta<\lambda_{D}\left(C_{1}^{\circ}, \ldots, C_{m}^{\circ}\right)$, where the first inequality follows from the implication (4.9) $\Rightarrow(4.10)$ in Theorem 4.1 and the second inequality is (4.17). Since $\delta>0$ is arbitrary, letting $\delta \rightarrow 0^{+}$in (4.18) yields (4.16).

The above theorem can be considered as a generalization and refinement of the duality between the normal property and property $(\mathrm{G})$ for two closed convex cones 
established by Jameson [26]. Recall that the collection $\left\{C_{1}, \ldots, C_{m}\right\}$ is said to have property $(\mathbf{G})$ if there exists $\rho>0$ such that

$$
\left(\sum_{i=1}^{m} C_{i}\right) \bigcap B_{H} \subset \sum_{i=1}^{m}\left(C_{i} \cap \frac{1}{\rho} B_{H}\right) .
$$

Actually, Jameson's definition was for the particular case when $m=2$ and the $C_{i}$ are closed convex cones. Property (G) has its origin in M. Krein's work. In 1940, M. Krein proved that if $K$ is a normal cone (i.e., $\{K,-K\}$ has the normal property and $K \cap(-K)=\{0\}$ ), then $K^{\ominus}$ is a generating cone (i.e., $K^{\ominus}-K^{\ominus}=H$ ) (see [35. Chapter V, Section 3, Subsection 3.2, Lemma 1]). Jameson's duality theory between property $(\mathrm{N})$ and property $(\mathrm{G})$ can be considered as an extension of M. Krein's work to two arbitrary closed convex cones. The following theorem shows that property $(\mathrm{G})$ for closed convex cones is actually a special case of the dual normal property 4.9).

Theorem 4.3. Let $K_{1}, \ldots, K_{m}$ be convex cones in $H$. Then

$$
\lambda_{D}\left(K_{1}, \ldots, K_{m}\right) \leq \lambda_{G}\left(K_{1}, \ldots, K_{m}\right) \leq m \cdot \lambda_{D}\left(K_{1}, \ldots, K_{m}\right),
$$

where $\lambda_{G}\left(K_{1}, \ldots, K_{m}\right)$ is a quantitative measurement of property $(\mathrm{G})$ for $\left\{K_{1}, \ldots\right.$, $\left.K_{m}\right\}$; namely,

$$
\lambda_{G}\left(K_{1}, \ldots, K_{m}\right):=\sup \left\{\rho \geq 0 \mid\left(\sum_{i=1}^{m} K_{i}\right) \cap B_{H} \subset \sum_{i=1}^{m}\left(K_{i} \cap \frac{1}{\rho} B_{H}\right)\right\},
$$

where $\frac{1}{\rho} B_{H}:=H$ if $\rho=0$.

Proof. By Lemma 4.1(3) and Lemma 2.19), we obtain that

$$
B_{H} \# \operatorname{co}\left(\bigcup_{i=1}^{m} K_{i}\right) \subset \operatorname{co}\left(\bigcup_{i=1}^{m}\left(K_{i} \# \frac{1}{\varepsilon} B_{H}\right)\right)
$$

is equivalent to

$$
B_{H} \cap\left(\sum_{i=1}^{m} K_{i}\right) \subset \operatorname{co}\left(\bigcup_{i=1}^{m}\left(K_{i} \cap \frac{1}{\varepsilon} B_{H}\right)\right) .
$$

By Lemma 2.1(9) and the fact that $m \cdot \operatorname{co}\left(S_{1}\right)=\operatorname{co}\left(m \cdot S_{1}\right)$ and $m\left(S_{1} \cap S_{2}\right)=$ $\left(m S_{1}\right) \cap\left(m S_{2}\right)$ for any sets $S_{1}$ and $S_{2}$, we obtain that

$$
\operatorname{co}\left(\bigcup_{i=1}^{m}\left(K_{i} \cap \frac{1}{\varepsilon} B_{H}\right)\right) \subset \sum_{i=1}^{m}\left(K_{i} \cap \frac{1}{\varepsilon} B_{H}\right) \subset \operatorname{co}\left(\bigcup_{i=1}^{m}\left(K_{i} \cap \frac{m}{\varepsilon} B_{H}\right)\right) .
$$

If $\lambda_{D}\left(K_{1}, \ldots, K_{m}\right)=0$, then $\lambda_{D}\left(K_{1}, \ldots, K_{m}\right) \leq \lambda_{G}\left(K_{1}, \ldots, K_{m}\right)$. Otherwise, for any $0<\varepsilon<\lambda_{D}\left(K_{1}, \ldots, K_{m}\right)$, (4.21) holds, which implies (4.22). By the first inclusion in (4.23), (4.22) implies that (4.19) holds with $\rho=\varepsilon$ and $C_{i}=K_{i}$. Hence,

$$
\varepsilon \leq \lambda_{G}\left(K_{1}, \ldots, K_{m}\right) \text { for all } 0<\varepsilon<\lambda_{D}\left(K_{1}, \ldots, K_{m}\right),
$$

which implies

$$
\lambda_{D}\left(K_{1}, \ldots, K_{m}\right) \leq \lambda_{G}\left(K_{1}, \ldots, K_{m}\right) .
$$


Conversely, if $\lambda_{G}\left(K_{1}, \ldots, K_{m}\right)=0$, then $\lambda_{G}\left(K_{1}, \ldots, K_{m}\right) \leq m \cdot \lambda_{D}\left(K_{1}, \ldots, K_{m}\right)$. Otherwise, for any $0<\rho<\lambda_{G}\left(K_{1}, \ldots, K_{m}\right)$,

$$
\left(\sum_{i=1}^{m} K_{i}\right) \bigcap B_{H} \subset \sum_{i=1}^{m}\left(K_{i} \cap \frac{1}{\rho} B_{H}\right) .
$$

By the second inclusion in (4.23), (4.25) implies that (4.22) holds with $\varepsilon=\rho / m$. Hence, (4.21) holds with $\varepsilon=\rho / m$, which implies

$$
\frac{\rho}{m} \leq \lambda_{D}\left(K_{1}, \ldots, K_{m}\right) \text { for all } 0<\rho<\lambda_{G}\left(K_{1}, \ldots, K_{m}\right),
$$

i.e.,

$$
\lambda_{G}\left(K_{1}, \ldots, K_{m}\right) \leq m \cdot \lambda_{D}\left(K_{1}, \ldots, K_{m}\right) .
$$

The required inequality (4.20) follows from (4.24) and (4.26). This completes the proof of Theorem 4.3

The above theorem shows that the dual normal property is equivalent to property $(\mathrm{G})$ for convex cones. However, for general convex sets, it is not clear whether property $(\mathrm{G})$ is related to the dual normal property or not.

An immediate consequence of the above two theorems is a generalization of Jameson's duality relationship between the normal property and property $(\mathrm{G})$ for closed convex cones.

Corollary 4.1. Let $K_{1}, \ldots, K_{m}$ be convex cones in $H$. Then $\left\{K_{1}, \ldots, K_{m}\right\}$ has the normal property if and only if $\left\{K_{1}, \ldots, K_{m}\right\}$ has the closed intersection property and $\left\{K_{1}^{\circ}, \ldots, K_{m}^{\circ}\right\}$ has property $(\mathrm{G})$.

Proof. Suppose that $\left\{K_{1}, \ldots, K_{m}\right\}$ has the normal property. By Theorem 3.1(3), it has the uniform normal property. It follows from Theorem $3.1(2)$ that $\left\{K_{1}, \ldots, K_{m}\right\}$ has the closed intersection property. Thus, by Theorems 4.2 and 4.3 , we have

$$
\lambda_{N}\left(K_{1}, \ldots, K_{m}\right) \leq \lambda_{G}\left(K_{1}^{\circ}, \ldots, K_{m}^{\circ}\right) \leq m \cdot \lambda_{N}\left(K_{1}, \ldots, K_{m}\right),
$$

which implies that $\left\{K_{1}, \ldots, K_{m}\right\}$ has the normal property (i.e., $\lambda_{N}\left(K_{1}, \ldots, K_{m}\right)>$ $0)$ if and only if $\left\{K_{1}^{\circ}, \ldots, K_{m}^{\circ}\right\}$ has property $(\mathrm{G})$ (i.e., $\lambda_{G}\left(K_{1}^{\circ}, \ldots, K_{m}^{\circ}\right)>0$ ).

\section{Strong CHIP, NORMal CHIP, AND WEAK NORMality}

Strong CHIP is the geometric version of the basic constraint qualification in convex optimization. The main result in this section is to show that the strong CHIP can be characterized by the weak normal property of the feasible direction cones. Simpler characterizations of the strong CHIP for closed convex cones will also be derived. We begin by defining some possible "conical hull intersection properties" that a collection of convex sets might possess.

Definition 5.1. Let $C_{1}, \ldots, C_{m}$ be convex subsets of $H$ having a nonempty intersection $C:=\bigcap_{1}^{m} C_{i}$.

(1) $\left\{C_{1}, \ldots, C_{m}\right\}$ is said to have the conical hull intersection property (CHIP) at $x \in C$ if the collection of convex cones

$$
\left\{\operatorname{con}\left(C_{1}-x\right), \ldots, \operatorname{con}\left(C_{m}-x\right)\right\}
$$


has the closed intersection property, i.e., if

$$
\overline{\bigcap_{1}^{m} \operatorname{con}\left(C_{i}-x\right)}=\bigcap_{1}^{m} \overline{\operatorname{con}}\left(C_{i}-x\right) .
$$

We say that $\left\{C_{1}, \ldots, C_{m}\right\}$ has the CHIP if it has the CHIP for each $x \in C$. (2) $\left\{C_{1}, \ldots, C_{m}\right\}$ is said to have the strong conical hull intersection property (strong CHIP) at $x \in C$ if

$$
\left(\bigcap_{i=1}^{m} C_{i}-x\right)^{\ominus}=\sum_{i=1}^{m}\left(C_{i}-x\right)^{\ominus} .
$$

We say that $\left\{C_{1}, \ldots, C_{m}\right\}$ has the strong CHIP if it has the strong CHIP for each $x \in C$.

(3) $\left\{C_{1}, \ldots, C_{m}\right\}$ is said to have the normal conical hull intersection property (normal CHIP) at $x \in C$ if the collection of convex cones

$$
\left\{\operatorname{con}\left(C_{1}-x\right), \ldots, \operatorname{con}\left(C_{m}-x\right)\right\}
$$

has the normal property. We say that $\left\{C_{1}, \ldots, C_{m}\right\}$ has the normal CHIP if it has the normal CHIP for each $x \in C$.

Remark 5.1. (a) While the normal CHIP property is new, the terminology "CHIP" was introduced in [11] and used in [12] for reformulations of certain constrained approximation problems in a Hilbert space. The "strong CHIP" was introduced in [18] (see also [19]) because it was noted there that the strong CHIP, rather than the CHIP, was the fundamental property which governed such problems. Indeed, it was shown in [15] that the strong CHIP was the precise property for which a KarushKuhn-Tucker (or Lagrange multiplier) condition was necessary and sufficient for characterizing an optimal solution to the problem of minimizing any continuous convex function $f: \bigcap_{1}^{m} C_{i} \rightarrow \mathbb{R}$ under convex constraints. In addition, in [17] it was shown that a natural generalization of a certain dual optimization problem over $\bigcap_{1}^{m} C_{i}$ has a solution if and only if $\left\{C_{1}, \ldots, C_{m}\right\}$ has the strong CHIP.

(b) It was shown in [19. (see also [16, Lemma 10.3, p. 239]) that $\left\{C_{1}, \ldots, C_{m}\right\}$ has the strong CHIP at $x$ if and only if it has the CHIP at $x$ and $\sum_{1}^{m}\left(C_{i}-x\right)^{\ominus}$ is closed.

(c) $\left\{C_{1}, \ldots, C_{m}\right\}$ has the normal CHIP at $x \in C$ if and only if the normality constant (cf. Definition 4.2) for $\left\{\operatorname{con}\left(C_{1}-x\right), \ldots, \operatorname{con}\left(C_{m}-x\right)\right\}$ is positive. In the next section, we will show that the normality constant for $\left\{\operatorname{con}\left(C_{1}-x\right), \ldots, \operatorname{con}\left(C_{m}-x\right)\right\}$ is bounded away from 0 uniformly over all $x \in C$ if and only if $\left\{C_{1}, \ldots, C_{m}\right\}$ has the linear regular property (cf. Theorems 6.1] and 6.2).

We can now exhibit a hierarchy among the various conical hull intersection properties.

Theorem 5.1. Let $C_{1}, \ldots, C_{m}$ be closed convex subsets of $H$ with a nonempty intersection $C:=\bigcap_{1}^{m} C_{i}$. For a given $x \in C$, consider the following statements:

(1) $\left\{C_{1}, \ldots, C_{m}\right\}$ has the normal CHIP at $x$.

(2) $\left\{C_{1}, \ldots, C_{m}\right\}$ has the strong CHIP at $x$.

(3) $\left\{C_{1}, \ldots, C_{m}\right\}$ has the CHIP at $x$.

Then $(1) \Rightarrow(2) \Rightarrow(3)$. 
Proof. By Remark 5.1(b) it follows that $(2) \Rightarrow(3)$. To prove that $(1) \Rightarrow(2)$, suppose that $\left\{C_{1}, \ldots, C_{m}\right\}$ has the normal CHIP at $x$. By Theorem 3.1(4) with $K_{i}:=\operatorname{con}\left(C_{i}-x\right)$, it follows that $\left\{\operatorname{con}\left(C_{1}-x\right), \ldots, \operatorname{con}\left(C_{m}-x\right)\right\}$ has the closed intersection property. By Remark 5.1(b), it follows that it suffices to verify that $\sum_{1}^{m} K_{i}^{\circ}$ is closed. It has been proved in Corollary 4.1 that $\left\{K_{1}^{\circ}, \ldots, K_{m}^{\circ}\right\}$ has the property $(\mathrm{G})$. Thus, $\sum_{1}^{m} K_{i}^{\circ}$ is closed by Lemma 5 in [26] (see also Lemma 2.8 in [9]). This completes the proof.

Remark 5.2. In general, $(3) \nRightarrow(2)[10$ and $(2) \nRightarrow(1)[3$ ] (see also [10]). If $\operatorname{dim} H \leq$ 3 , it was proved that $(1) \Leftrightarrow(2)$ (see [1]) and $(2) \Leftrightarrow(3)$ (see [10]).

By a duality analysis of the weak normal property, we can prove that the strong CHIP is equivalent to the weak normal property for convex cones. The key step in the proof of this equivalence relationship is to establish the following dual form of the weak normal property.

Theorem 5.2. (Dual weak normality) Let $C_{1}, \ldots, C_{m}$ be convex sets in $H$ with nonempty intersection $C$. If $\left\{C_{1}, \ldots, C_{m}\right\}$ has the weak normal property, then for any $y \in H$, there exists $\varepsilon(y)>0$ such that

$$
[0, y] \# C^{\circ} \subset \operatorname{co}\left[\bigcup_{i=1}^{m}\left(\frac{1}{\varepsilon(y)} B_{H} \# C_{i}^{\circ}\right)\right],
$$

where $[0, y]:=\{\theta y \mid 0 \leq \theta \leq 1\})$. Conversely, if (5.3) holds and $\left\{C_{1}, \ldots, C_{m}\right\}$ has the closed intersection property, then $\left\{C_{1}, \ldots, C_{m}\right\}$ has the weak normal property.

Proof. By Definition 3.1 (3), $\left\{C_{1}, \ldots, C_{m}\right\}$ has the weak normal property if and only if for each $y \in H$ there exists $\varepsilon(y)>0$ such that

$$
\bigcap_{i=1}^{m}\left[C_{i}+\varepsilon(y) B_{H}\right] \subset C+\{y\}^{\circ} .
$$

Taking the polar on both sides of (5.4), we obtain

$$
[0, y] \# C^{\circ}=\left(C+\{y\}^{\circ}\right)^{\circ} \subset\left(\bigcap_{i=1}^{m}\left[C_{i}+\varepsilon(y) B_{H}\right]\right)^{\circ}=\operatorname{co}\left[\bigcup_{i=1}^{m}\left(\frac{1}{\varepsilon(y)} B_{H} \# C_{i}^{\circ}\right)\right],
$$

where the first equality follows by Lemma 4.1(7), the inclusion follows from (5.4), and the last equality is by Lemma 4.2. This proves the first statement in Theorem 5.2 .

Conversely, assume (5.3) holds. By calculating the polar form of (5.3), we get

$$
\begin{aligned}
& \overline{\bigcap_{i=1}^{m}\left(C_{i}+\frac{\varepsilon(y)}{m} B_{H}\right)}=\left[\bigcap_{i=1}^{m}\left(C_{i}+\frac{\varepsilon(y)}{m} B_{H}\right)\right]^{\circ}=\left[\bigcup_{i=1}^{m}\left(\frac{m}{\varepsilon(y)} B_{H} \# C_{i}^{\circ}\right)\right]^{\circ} \\
= & \left(\operatorname{co}\left[\bigcup_{i=1}^{m}\left(\frac{m}{\varepsilon(y)} B_{H} \# C_{i}^{\circ}\right)\right]\right)^{\circ} \subset\left([0, y] \# C^{\circ}\right)^{\circ}=\left(C+\{y\}^{\circ}\right)^{\circ \circ}=\overline{C+\{y\}^{\circ}}
\end{aligned}
$$

where the first equality and the last equality are by Lemma [2.1]4), the second equality follows from Lemma 4.2, the third equality is by Lemma 2.1(6), the inclusion follows from (5.3), and the fourth equality is by Lemma 4.1(7). Applying 
Lemma 2.4 with $\varepsilon=\varepsilon(y) / m$ and $D=C+\{y\}^{\circ}$, we obtain

$$
\bigcap_{i=1}^{m}\left(C_{i}+\frac{\varepsilon(y)}{2 m} B_{H}\right) \subset C+\{y\}^{\circ} .
$$

Thus, $\left\{C_{1}, \ldots, C_{m}\right\}$ has the weak normal property. This completes the proof of Theorem 5.3.

Using Theorem 5.2 we can prove that the strong CHIP is a dual form of the weak normality property for convex cones. This leads to the following characterization of the strong CHIP via the weak normality of feasible direction cones.

Theorem 5.3. Let $C_{1}, \ldots, C_{m}$ be closed convex subsets of $H$ with nonempty intersection $C$, and let $x \in C$. Then $\left\{C_{1}, \ldots, C_{m}\right\}$ has the strong CHIP at $x$ if and only if the collection of feasible direction cones $\left\{\operatorname{con}\left(C_{1}-x\right), \ldots, \operatorname{con}\left(C_{m}-x\right)\right\}$ has the weak normal property.

Proof. Let $K_{i}:=\operatorname{con}\left(C_{i}-x\right)$ and $K:=\operatorname{con}(C-x)$. Since $x \in C \subset C_{i}$, it follows that $K_{i}^{\circ}=\left(C_{i}-x\right)^{\ominus}$ and $K^{\circ}=(C-x)^{\ominus}$. Thus, the strong CHIP of $\left\{C_{1}, \ldots, C_{m}\right\}$ is equivalent to $K^{\circ}=\sum_{i=1}^{m} K_{i}^{\circ}$. Since $K_{i}^{\circ} \subset K^{\circ}$, we obtain that $\left\{C_{1}, \ldots, C_{m}\right\}$ has the strong CHIP if and only if

$$
K^{\circ} \subset \sum_{i=1}^{m} K_{i}^{\circ}
$$

Therefore, it suffices to prove that (5.8) holds if and only if $\left\{K_{1}, \ldots, K_{m}\right\}$ has the weak normal property.

By Theorem 5.2 the weak normal property of $\left\{K_{1}, \ldots, K_{m}\right\}$ implies that for any $y \in H$, there exists $\varepsilon(y)>0$ such that

$$
[0, y] \# K^{\circ} \subset \operatorname{co}\left[\bigcup_{i=1}^{m}\left(\frac{1}{\varepsilon(y)} B_{H} \# K_{i}^{\circ}\right)\right] .
$$

By Lemma 4.1(3), (5.9) can be rewritten as

$$
[0, y] \cap K^{\circ} \subset \operatorname{co}\left[\bigcup_{i=1}^{m}\left(\frac{1}{\varepsilon(y)} B_{H} \cap K_{i}^{\circ}\right)\right] .
$$

It follows from (5.10) and Lemma 2.1 (9) that

$$
[0, y] \cap K^{\circ} \subset \sum_{i=1}^{m}\left(\frac{1}{\varepsilon(y)} B_{H} \cap K_{i}^{\circ}\right) .
$$

For any $y \in K^{\circ}$, (5.11) implies $y \in \sum_{i=1}^{m} K_{i}^{\circ}$. Thus, (5.8) holds.

On the other hand, if (5.8) holds, then for $y \in K^{\circ}$, there exist $y_{i} \in K_{i}^{\circ}$ such that $y=\sum_{i=1}^{m} y_{i}$. Let $\varepsilon(y)>0$ be such that $\left\|m y_{i}\right\|<1 / \varepsilon(y)$. Then $y=\frac{1}{m} \sum_{i=1}^{m}\left(m y_{i}\right) \in$ co $\left[\bigcup_{i=1}^{m}\left(\frac{1}{\varepsilon(y)} B_{H} \cap K_{i}^{\circ}\right)\right]$. Hence (5.10) holds. If $y \notin K^{\circ}$, then $[0, y] \cap K^{\circ}=\{0\}$ and (5.10) holds for any $\varepsilon(y)>0$. By Lemma 4.1(3), (5.9) holds for any $y \in H$. By $(2) \Rightarrow(3)$ in Theorem 5.1 the strong CHIP of $\left\{C_{1}, \ldots, C_{m}\right\}$ at $x$ implies that $\left\{K_{1}, \ldots, K_{m}\right\}$ has the closed intersection property. By Theorem $5.2,\left\{K_{1}, \ldots, K_{m}\right\}$ has the weak normal property. This completes the proof of Theorem5.3. 
Remark 5.3. (a) From the proof of Theorem [5.3, we know that the weak normal property is a dual characterization of the strong CHIP. In contrast, there are some obvious alternative ways of describing the strong CHIP. The following statements are equivalent for any $x \in \bigcap_{1}^{m} C_{i}[18$ p. 389]:

(1) $\left\{C_{1}, \ldots, C_{m}\right\}$ has the strong CHIP at $x$;

(2) $N_{\left(\cap_{1}^{m} C_{i}\right)}(x)=\sum_{1}^{m} N_{C_{i}}(x)$;

(3) $\partial I_{\left(\cap_{1}^{m} C_{i}\right)}(x)=\sum_{1}^{m} \partial I_{C_{i}}(x)$.

Here $I_{D}(x)$ denotes the indicator function of a set $D$, which is defined on $H$ by $I_{D}(x)=0$ if $x \in D$ and $I_{D}(x)=+\infty$ if $x \notin D$, and $\partial I_{D}(x)$ is the subdifferential of $I_{D}$ at $x$, which is defined by

$$
\partial I_{D}(x):=\left\{z \in H \mid\langle z, y-x\rangle+I_{D}(x) \leq I_{D}(y) \text { for every } y \in H\right\} .
$$

(b) The relation (2) may be regarded as the geometric version of the basic constraint qualification. In fact, let $g_{i}$ be a convex function on $H$ for $i=1,2, \ldots, m$, and let $C_{i}=\left\{x \in H \mid g_{i}(x) \leq 0\right\}$ and suppose that there exists an $x_{0} \in H$ such that $g_{i}\left(x_{0}\right)<0$ for $i=1,2, \ldots, m$. That is, the "Slater condition" holds. Then for $x \in C_{i}$, we have

$$
N_{C_{i}}(x)= \begin{cases}\left\{\theta y \mid y \in \partial g_{i}(x), \theta \geq 0\right\} & \text { if } g_{i}(x)=0, \\ \{0\} & \text { if } g_{i}(x)<0 .\end{cases}
$$

It was observed in [17] that if int $\bigcap_{1}^{m} C_{i} \neq \emptyset$, then $\left\{C_{1}, \ldots, C_{m}\right\}$ has the strong CHIP. But the Slater condition implies the nonemptiness of int $\bigcap_{1}^{m} C_{i}$. Thus, by (5.12), we can rewrite (2) as

$$
N_{\left(\cap_{1}^{m} C_{i}\right)}(x)=\left\{\sum_{i \in I(x)} \theta_{i} y_{i} \mid y_{i} \in \partial g_{i}(x), \theta_{i} \geq 0\right\},
$$

where $I(x):=\left\{i \mid g_{i}(x)=0\right\}$ is the active index set at $x$ and the scalars $\theta_{i}$ in (5.13) are called Lagrange multipliers.

(c) Li, Nahak, and Singer 32 gave a characterization of the basic constraint qualification for convex inequalities $g_{i}(x) \leq 0(1 \leq i \leq m)$ in terms of upper semicontinuity of certain cone mappings. It would be interesting to know whether Theorem 2.4 in [32] can be reformulated to give a characterization of the strong CHIP for $\left\{C_{1}, \ldots, C_{m}\right\}$.

Our next result shows that, in the case of convex cones, having the strong or normal CHIP is equivalent to having this property at the single point zero.

Theorem 5.4. Let $K_{1}, \ldots, K_{m}$ be convex cones. Then $\left\{K_{1}, \ldots, K_{m}\right\}$ has the strong CHIP or the normal CHIP if and only if it has the same property at zero.

Proof. Let $K:=\bigcap_{i=1}^{m} K_{i}$. Suppose now that $\left\{K_{1}, \ldots, K_{m}\right\}$ has the strong CHIP at 0 . By Theorem 5.3. $\left\{K_{1}, \ldots, K_{m}\right\}$ has the weak normal property, i.e., for every $y \in H$, there exists $\varepsilon(y)>0$ such that

$$
\bigcap_{i=1}^{m}\left(K_{i}+\varepsilon(y) B_{H}\right) \subset K+\{y\}^{\circ} .
$$

Lemma 2.5] and (5.14) imply that

$$
\bigcap_{i=1}^{m}\left[\operatorname{con}\left(K_{i}-x\right)+\varepsilon(y) B_{H}\right] \subset \bigcap_{i=1}^{m} \operatorname{con}\left(K_{i}-x\right)+\{y\}^{\circ} \text { for all } x \in K .
$$


Applying Theorem 5.3 with $C_{i}=K_{i}$, we get that $\left\{K_{1}, \ldots, K_{m}\right\}$ has the strong CHIP at $x$ for $x \in K$, i.e., $\left\{K_{1}, \ldots, K_{m}\right\}$ has the strong CHIP.

Suppose that $\left\{K_{1}, \ldots, K_{m}\right\}$ has the normal CHIP at 0 . This means that $\left\{K_{1}, \ldots\right.$, $\left.K_{m}\right\}$ has the normal property, i.e., there exists $\varepsilon>0$ such that

$$
\bigcap_{i=1}^{m}\left(K_{i}+\varepsilon B_{H}\right) \subset \bigcap_{i=1}^{m} K_{i}+B_{H} .
$$

Using Lemma 2.5, (5.16) implies that

$$
\bigcap_{i=1}^{m}\left[\operatorname{con}\left(K_{i}-x\right)+\varepsilon B_{H}\right] \subset \bigcap_{i=1}^{m} \operatorname{con}\left(K_{i}-x\right)+B_{H} \text { for all } x \in K .
$$

This proves that $\left\{\operatorname{con}\left(K_{1}-x\right), \ldots, \operatorname{con}\left(K_{m}-x\right)\right\}$ has the normal property. In other words, $\left\{K_{1}, \ldots, K_{m}\right\}$ has the normal CHIP at arbitrary $x \in K$, which was to be proved.

Remark 5.4. A proof that a set of closed convex cones has the strong CHIP at zero if and only if it has the strong CHIP was first given in [9].

The following characterizations of the strong CHIP for convex cones can be derived immediately from Theorems 5.3 and 5.4 .

Corollary 5.1. Let $K_{1}, \ldots, K_{m}$ be convex cones. Then the following statements are equivalent:

(1) $\left\{K_{1}, \ldots, K_{m}\right\}$ has the strong CHIP;

(2) $\left\{K_{1}, \ldots, K_{m}\right\}$ has the weak normal property;

(3) $\left(\bigcap_{i=1}^{m} K_{i}\right)^{\ominus}=\sum_{i=1}^{m} K_{i}^{\ominus}$.

Moreover, if $K_{1}, \ldots, K_{m}$ are closed, these statements are equivalent to the following statement:

(4) $\sum_{1}^{m} K_{i}^{\ominus}$ is closed.

Proof. Since the relation (3) is just the statement that $\left\{K_{1}, \ldots, K_{m}\right\}$ has the strong CHIP at 0 , the equivalence of (1) and (3) follows from Theorem 5.4. Taking $x=0$ and $C_{i}=K_{i}$ in Theorem 5.3 yields the equivalence of (2) and (3). Since dual cones are always closed, (4) is an obvious consequence of (3). Finally, if the $K_{i}$ are closed and (4) holds, then by Lemma 2.1(5), we have that $\left(\bigcap_{1}^{m} K_{i}\right)^{\ominus}=\overline{\sum_{1}^{m} K_{i}^{\ominus}}=$ $\sum_{1}^{m} K_{i}^{\ominus}$. That is, (3) holds.

Remark 5.5. Some authors have called the equation in Corollary 5.1(3) the MoreauRockafellar equality for convex cones in honor of the two mathematicians who have established the first sufficient condition for the validity of this equation (see, e.g., 24, 27 28]). The problem of getting a dual description of the Moreau-Rockafellar equality has attracted the attention of many mathematicians (see the survey [2]).

When applied to closed linear subspaces, in addition to what we have in Corollary 5.1, there is another interesting characterization of the strong CHIP in terms of the projections onto the orthogonal complements of these subspaces.

Corollary 5.2. Let $M_{1}, \ldots, M_{m}$ be closed linear subspaces in $H$, and let $M=$ $\bigcap_{1}^{m} M_{i}$. Then the following statements are equivalent:

(1) $\left\{M_{1}, \ldots, M_{m}\right\}$ has the strong CHIP;

(2) $\left\{M_{1}, \ldots, M_{m}\right\}$ has the weak normal property; 
(3) $M^{\perp}=\sum_{1}^{m} M_{i}^{\perp}$;

(4) $\sum_{1}^{m} M_{i}^{\perp}$ is closed;

(5) $\left\|P_{M_{m}} P_{M_{m-1}} \cdots P_{M_{1}} P_{M^{\perp}}\right\|<1$.

Proof. The equivalence of the first four statements is an immediate consequence of Corollary 5.1 The equivalence of the last two statements follows from a combination of results from [6, Lemma 5.18 and Theorem 5.19] and [8, Lemma 3.7.3 and Theorem 3.7.4] (see also 4, Theorem 5.5.1]).

Remark 5.6. (a) One can find some historical motivation for studying the strong CHIP (or Moreau-Rockafellar equality) for two closed linear subspaces in 2 .

(b) One reason for the possible interest in this corollary stems from the fact that it shows for the first time a close connection between "fast" convergence of the method of alternating projections and the strong conical hull intersection property. More precisely, the well-known von Neumann-Halperin method of alternating projections can be stated as follows: If $M_{i}$ and $M$ are given as in Corollary 5.2, then

$$
\lim _{n}\left(P_{M_{m}} P_{M_{m-1}} \cdots P_{M_{1}}\right)^{n}(x)=P_{M}(x)
$$

for each $x \in H$ (see, e.g., [16] Corollary 9.28, p. 217]). Moreover, concerning the rate of convergence of this method, it is not difficult to show (see [16, Lemma 9.30, p. 218]) that

$$
\begin{aligned}
\left.\| P_{M_{m}} P_{M_{m-1}} \cdots P_{M_{1}}\right)^{n}-P_{M} \|= & \left\|\left(P_{M_{m}} P_{M_{m-1}} \cdots P_{M_{1}} P_{M^{\perp}}\right)^{n}\right\| \\
& \leq\left\|P_{M_{m}} P_{M_{m-1}} \cdots P_{M_{1}} P_{M^{\perp}}\right\|^{n} .
\end{aligned}
$$

It follows from this and the above corollary that when the collection of subspaces has the strong CHIP, then the rate of convergence in the method of alternating projections is geometric. We should mention that without some additional condition on the subspaces, the convergence may be arbitrarily slow (see [21]).

\section{LINEAR REGULARITY AND NORMALITY}

The main purpose of this section is to show that linear regularity can be characterized by normality of certain families of the convex sets generated by scaling and/or shifting of the original collection of convex sets. In particular, we will show that linear regularity is equivalent to uniform normality. Moreover, linear regularity can also be characterized by the fact that the normality constant for the feasible direction cones $\left\{\operatorname{con}\left(C_{1}-x\right), \ldots, \operatorname{con}\left(C_{m}-x\right)\right\}$ is bounded away from 0 uniformly over all $x$ in the intersection $\bigcap_{1}^{m} C_{i}$.

First we give the formal definitions of (bounded) linear regularity for a collection of convex sets in the Hilbert space $H$.

Definition 6.1. Let $C_{1}, \ldots, C_{m}$ be convex subsets of $H$ with $C:=\bigcap_{1}^{m} C_{i} \neq \emptyset$.

(1) $\left\{C_{1}, \ldots, C_{m}\right\}$ is said to have the bounded linear regularity property if for any constant $\rho>0$, there exists a constant $\gamma_{\rho}$ such that

$$
d(x, C) \leq \gamma_{\rho} \max _{1 \leq i \leq m} d\left(x, C_{i}\right) \quad \text { whenever }\|x\| \leq \rho .
$$

(2) $\left\{C_{1}, \ldots, C_{m}\right\}$ is said to have the linear regularity property if there exists a constant $\gamma$ such that

$$
d(x, C) \leq \gamma \max _{1 \leq i \leq m} d\left(x, C_{i}\right) \quad \text { for every } x \in H .
$$


Remark 6.1. (a) The linear regularity of closed convex sets was first introduced by Bauschke and Borwein [5] to quantify the relationship between the distance from an approximate solution $x$ to the feasible set $\bigcap_{i} C_{i}$ and the distance from $x$ to each individual constraint set $C_{i}$.

(b) The linear regularity property is the geometric version of the so-called Hoffman's error bound for approximate solutions of convex inequalities $g_{i}(x) \leq 0$ $(1 \leq i \leq m)$ :

$$
d(x, C) \leq \gamma \max _{1 \leq i \leq m} \max \left\{g_{i}(x), 0\right\} \text { for all } x \in H,
$$

where $C_{i}:=\left\{z \in H: g_{i}(z) \leq 0\right\}$ and $C:=\bigcap_{i=1}^{m} C_{i}$. In the case that each $g_{i}(x)$ is an affine function, [6.3) was proved by Hoffman [23. In this case, the gradient $\nabla g_{i}(x) \equiv a_{i}$ is a constant vector, and it is easy to verify that $d\left(x, C_{i}\right)=$ $\frac{1}{\left\|a_{i}\right\|} \max \left\{g_{i}(x), 0\right\}$. Therefore, if each $C_{i}$ is defined by an affine inequality $g_{i}(x) \leq 0$, then the linear regularity of $\left\{C_{1}, \ldots, C_{m}\right\}$ is the same as Hoffman's error bound (6.3) for approximate solutions of affine inequalities $g_{i}(x) \leq 0(1 \leq i \leq m)$.

(c) In general, (bounded) linear regularity is defined for closed convex sets. If $C_{1}, \ldots, C_{m}$ are not closed, then (bounded) linear regularity implies that they have the closed intersection property. In fact, if $x \in \bigcap_{i=1}^{m} \overline{C_{i}}$, then $d\left(x, C_{i}\right)=0$ for $1 \leq i \leq m$. By either (6.1) or (6.2), we have $d(x, C)=0$, i.e., $x \in \bar{C}$. This proves the closed intersection property of $\left\{C_{1}, \ldots, C_{m}\right\}$. By $d(x, S)=d(x, \bar{S})$ for any $x$ and $S$ in $H,\left\{\overline{C_{1}}, \ldots, \overline{C_{m}}\right\}$ has the (bounded) linear regularity property. Conversely, if $\left\{C_{1}, \ldots, C_{m}\right\}$ has the closed intersection property, then (bounded) linear regularity of $\left\{\overline{C_{1}}, \ldots, \overline{C_{m}}\right\}$ implies (bounded) linear regularity of $\left\{C_{1}, \ldots, C_{m}\right\}$.

(d) When $H$ is a finite-dimensional Euclidean space, the bounded linear regularity is equivalent to the so-called metric regularity. Recall that $\left\{C_{1}, \ldots, C_{m}\right\}$ is said to be metrically regular at $x \in \bigcap_{1}^{m} C_{i}$ if there exist positive constants $\delta_{x}$ and $\gamma_{x}$ such that

$$
d(y, C) \leq \gamma_{x} \max _{1 \leq i \leq m} d\left(y, C_{i}\right) \text { for all } y \in H \text { with }\|y-x\| \leq \delta_{x} .
$$

We say that $\left\{C_{1}, \ldots, C_{m}\right\}$ is metrically regular if it is metrically regular at every $x \in \bigcap_{1}^{m} C_{i}$. It is obvious that bounded linear regularity implies metric regularity. When $H$ is finite dimensional, by using the finite-covering theorem for a compact set, one can also prove that metric regularity implies bounded linear regularity (cf. 31, the proof of Theorem 8]).

(e) Suppose that $H$ is a finite-dimensional Euclidean space and $C_{i}:=\left\{x \mid g_{i}(x) \leq\right.$ $0\}$, where $g_{i}$ are differentiable convex functions on $H$. If $N_{C_{i}}(x)=\left\{t \nabla g_{i}(x)\right.$ $t \geq 0\}$ whenever $g_{i}(x)=0$, then the bounded linear regularity of $\left\{C_{1}, \ldots, C_{m}\right\}$ is equivalent to Abadie's constraint qualification or the standard basic constraint qualification for $g_{i}(x) \leq 0(1 \leq i \leq m)$ (cf. the proofs of [31, Theorems 8 and 10]). We leave the details to the interested reader.

Before proving the main results that characterize (bounded) linear regularity, we need the following technical lemma about normality and bounded linear regularity.

Lemma 6.1. Let $C_{1}, \ldots, C_{m}$ be closed convex subsets of $H$ with $0 \in C:=\bigcap_{i=1}^{m} C_{i}$. For fixed positive constants $\rho$, $\tau$, and $\gamma_{\rho}$, consider the following relations:

$$
d(x, C) \leq \gamma_{\rho} \max _{1 \leq i \leq m} d\left(x, C_{i}\right) \quad \text { whenever }\|x\| \leq \rho,
$$




$$
\begin{aligned}
& \left(\rho B_{H}\right) \cap\left[\bigcap_{i=1}^{m}\left(C_{i}+\frac{\delta}{\gamma_{\rho}} B_{H}\right)\right] \subset C+\delta B_{H} \quad \text { for every } \delta>0, \\
& \left(\rho B_{H}\right) \cap\left[\bigcap_{i=1}^{m}\left(C_{i}+\frac{\delta}{\gamma_{\rho}} B_{H}\right)\right] \subset C+\delta B_{H} \quad \text { for every } 0<\delta \leq \tau, \\
& d(x, C) \leq \gamma_{\rho} \max _{1 \leq i \leq m} d\left(x, C_{i}\right) \quad \text { whenever }\|x\| \leq \rho \text { and } d(x, C) \leq \frac{\tau}{\gamma_{\rho}}, \\
& \lambda_{N}\left(\operatorname{con}\left(C_{1}-x\right), \ldots, \operatorname{con}\left(C_{m}-x\right)\right) \geq \frac{1}{\gamma_{\rho}} \text { for all } x \in C \text { with }\|x\|<\rho .
\end{aligned}
$$

Then (6.4) $\Leftrightarrow(6.5) \Leftrightarrow(6.6) \Leftrightarrow(6.7) \Leftrightarrow$ (6.8).

Proof. (6.4) $\Rightarrow(6.5)$ Assume that (6.4) holds. Let $x$ be an element in the set on the left-hand side of (6.5). Then $\|x\| \leq \rho$ and

$$
d\left(x, C_{i}\right) \leq \frac{\delta}{\gamma_{\rho}} \text { for all } 1 \leq i \leq m
$$

It follows from (6.9) and (6.4) that

$$
d(x, C) \leq \gamma_{\rho} \max _{1 \leq i \leq m} d\left(x, C_{i}\right) \leq \gamma_{\rho} \cdot \frac{\delta}{\gamma_{\rho}}=\delta,
$$

which implies $x \in C+\delta B_{H}$. This proves that (6.5) holds.

(6.5) $\Rightarrow$ (6.4) Let $x \in H$ with $\|x\| \leq \rho$. Define $\delta:=\gamma_{\rho} \max _{1 \leq i \leq m} d\left(x, C_{i}\right)$. Then $x$ is in the set on the left-hand side of (6.5). It follows from (6.5) that $d(x, C) \leq \delta$, i.e., (6.4) holds.

(6.5) $\Rightarrow(6.6)$ This is obvious.

$(6.6) \Rightarrow(6.7)$ Assume that (6.6) holds. For any $\|x\| \leq \rho$ and $d(x, C) \leq \frac{\tau}{\gamma_{\rho}}$, we have for each $i$ that

$$
d\left(x, C_{i}\right) \leq d(x, C) \leq \frac{\tau}{\gamma_{\rho}}
$$

i.e., $\delta:=\gamma_{\rho} \max _{1 \leq i \leq m} d\left(x, C_{i}\right) \leq \tau$. Thus $x$ is an element in the set on the left-hand side of (6.6). By (6.6), we have $x \in C+\delta B_{H}$, i.e., (6.7) holds.

(6.7) $\Rightarrow$ (6.4) Assume that (6.7) holds. Let $x \in H$ with $\|x\| \leq \rho$. Since $C$ is a closed convex set, there is $x^{*} \in C$ such that $d(x, C)=\left\|x-x^{*}\right\|$. If $\left\|x-x^{*}\right\|>\frac{\tau}{\gamma_{\rho}}$, for $\theta:=\frac{\tau}{\gamma_{\rho} d(x, C)} \in(0,1)$, we have

$$
d\left(\hat{x}, C_{i}\right) \leq d(\hat{x}, C)=\theta \cdot d(x, C)=\frac{\tau}{\gamma_{\rho}},
$$

where $\hat{x}:=(1-\theta) x^{*}+\theta x$. Since $0 \in C$, the second statement in Lemma2.6 implies that $\left\|x^{*}\right\| \leq\|x\| \leq \rho$. Thus

$$
\|\hat{x}\| \leq(1-\theta)\left\|x^{*}\right\|+\theta\|x\| \leq \rho .
$$

By (6.10), (6.11), and (6.7), we get

$$
d(\hat{x}, C) \leq \gamma_{\rho} \max _{1 \leq i \leq m} d\left(\hat{x}, C_{i}\right) .
$$

Since $x^{*} \in C \subset C_{i}$, by the convexity of the distance function, we have

$$
\max _{1 \leq i \leq m} d\left(\hat{x}, C_{i}\right) \leq \max _{1 \leq i \leq m}\left[\theta \cdot d\left(x, C_{i}\right)+(1-\theta) \cdot d\left(x^{*}, C_{i}\right)\right]=\theta \max _{1 \leq i \leq m} d\left(x, C_{i}\right) .
$$


It follows from (6.10), 6.12), and (6.13) that

$$
d(x, C) \leq \gamma_{\rho} \max _{1 \leq i \leq m} d\left(x, C_{i}\right) .
$$

If $\left\|x-x^{*}\right\| \leq \frac{\tau}{\gamma_{\rho}}$, then (6.7) implies (6.14). As a consequence, (6.14) holds whenever $\|x\| \leq \rho$, i.e., (6.4) holds.

$(6.5) \Rightarrow(6.8)$ Subtracting $x$ from both sides of (6.5) and dividing the resulting inclusion by $\delta>0$, we get

$$
\begin{aligned}
& \frac{1}{\delta}\left(\rho B_{H}-x\right) \bigcap\left[\bigcap_{i=1}^{m}\left(\frac{1}{\delta}\left(C_{i}-x\right)+\frac{1}{\gamma_{\rho}} B_{H}\right)\right] \\
& \subset\left(\bigcap_{i=1}^{m} \frac{1}{\delta}\left(C_{i}-x\right)\right)+B_{H} \text { for all } x \in C \cap\left(\rho B_{H}\right) .
\end{aligned}
$$

We claim that (6.15) implies that

$$
\bigcap_{i=1}^{m}\left[\operatorname{con}\left(C_{i}-x\right)+\frac{1}{\gamma_{\rho}} B_{H}\right] \subset\left[\bigcap_{i=1}^{m} \operatorname{con}\left(C_{i}-x\right)\right]+B_{H} \text { for all } x \in C \text { with }\|x\|<\rho .
$$

In fact, if $y \in \bigcap_{i=1}^{m}\left[\operatorname{con}\left(C_{i}-x\right)+\frac{1}{\gamma_{\rho}} B_{H}\right]$, then there exist $\delta_{i}>0$ such that

$$
y \in \bigcap_{i=1}^{m}\left[\frac{1}{\delta_{i}}\left(C_{i}-x\right)+\frac{1}{\gamma_{\rho}} B_{H}\right] .
$$

Moreover, since $\|x\|<\rho$, there exists $\delta_{0}>0$ such that $y \in \frac{1}{\delta_{0}}\left(\rho B_{H}-x\right)$. As a consequence, $y$ is in the set on the left-hand side of (6.15) with $\delta:=\min \left\{\delta_{0}, \delta_{1}, \ldots, \delta_{m}\right\}$. It follows from (6.15) that

$$
y \in\left(\bigcap_{i=1}^{m} \frac{1}{\delta}\left(C_{i}-x\right)\right)+B_{H} \subset\left[\bigcap_{i=1}^{m} \operatorname{con}\left(C_{i}-x\right)\right]+B_{H},
$$

which proves (6.16). By the definition of the normality constant and (6.16), we obtain (6.8).

$(\underline{6.8}) \Rightarrow(6.4)$ Using the definition of the normality constant and (6.8), we obtain

$$
\begin{aligned}
& {\left[\bigcap_{i=1}^{m}\left(\operatorname{con}\left(C_{i}-x\right)+\frac{1}{\bar{\gamma}_{\rho}} B_{H}\right)\right]} \\
& \quad \subset\left(\bigcap_{i=1}^{m} \operatorname{con}\left(C_{i}-x\right)\right)+B_{H} \text { for all } x \in C \text { with }\|x\|<\rho,
\end{aligned}
$$

where $\bar{\gamma}_{\rho}>\gamma_{\rho}$. Note that

$$
\begin{aligned}
& \left(\bigcap_{i=1}^{m} \operatorname{con}\left(C_{i}-x\right)\right)+B_{H}=\left(\bigcap_{i=1}^{m} \bigcup_{\theta>0} \theta\left(C_{i}-x\right)\right)+B_{H} \\
& \quad=\left(\bigcup_{\theta>0} \bigcap_{i=1}^{m} \theta\left(C_{i}-x\right)\right)+B_{H}=\bigcup_{\theta>0}\left[\theta(C-x)+B_{H}\right]=\bigcup_{\theta>0} \theta\left(C-x+\frac{1}{\theta} B_{H}\right),
\end{aligned}
$$


where the second equality follows from Lemma 2.2. Similarly,

$$
\begin{aligned}
& \bigcap_{i=1}^{m}\left(\operatorname{con}\left(C_{i}-x\right)+\frac{1}{\bar{\gamma}_{\rho}} B_{H}\right)=\bigcap_{i=1}^{m}\left(\left[\bigcup_{\theta>0} \theta\left(C_{i}-x\right)\right]+\frac{1}{\bar{\gamma}_{\rho}} B_{H}\right) \\
& =\bigcap_{i=1}^{m} \bigcup_{\theta>0}\left(\theta\left(C_{i}-x\right)+\frac{1}{\bar{\gamma}_{\rho}} B_{H}\right)=\bigcup_{\theta>0}\left[\theta \bigcap_{i=1}^{m}\left(C_{i}-x+\frac{1}{\theta \bar{\gamma}_{\rho}} B_{H}\right)\right],
\end{aligned}
$$

where the third equality follows from Lemma 2.2 Therefore, (6.17) can be rewritten as

$$
\begin{aligned}
& \bigcup_{\theta>0}\left[\theta \bigcap_{i=1}^{m}\left(C_{i}-x+\frac{1}{\theta \bar{\gamma}_{\rho}} B_{H}\right)\right] \\
& \subset \bigcup_{\theta>0} \theta\left(C-x+\frac{1}{\theta} B_{H}\right) \text { for all } x \in C \text { with }\|x\|<\rho .
\end{aligned}
$$

Let $\delta>0$ and $\|y\|<\rho$ be such that

$$
y \in \bigcap_{i=1}^{m}\left(C_{i}+\frac{\delta}{\bar{\gamma}_{\rho}} B_{H}\right) .
$$

Since $C$ is closed, there is $y^{*} \in C$ such that $\left\|y-y^{*}\right\|=d(y, C)$. Then, by (6.19), we get that

$$
\frac{1}{\delta}\left(y-y^{*}\right) \in \frac{1}{\delta} \bigcap_{i=1}^{m}\left(C_{i}-y^{*}+\frac{\delta}{\bar{\gamma}_{\rho}} B_{H}\right) .
$$

Since $0 \in C$, by the second statement in Lemma 2.6, we have $\left\|y^{*}\right\|<\rho$. So (6.18) holds for $x=y^{*}$. That is, there exist $\theta^{*}>0, z \in C$, and $u \in B_{H}$ such that

$$
\frac{1}{\delta}\left(y-y^{*}\right)=\theta^{*}\left(z-y^{*}\right)+u
$$

which can be rewritten as follows:

$$
\frac{1}{\theta^{*} \delta}\left(y-y^{*}\right)=z+\frac{u}{\theta^{*}}-y^{*} .
$$

Since $d(y, C)=\left\|y-y^{*}\right\|$, by the first statement in Lemma 2.6, we have $y-y^{*} \in$ $\left(C-y^{*}\right)^{\ominus}$. By (6.21), it follows that $z+\frac{u}{\theta^{*}}-y^{*} \in\left(C-y^{*}\right)^{\ominus}$, which implies

$$
\left\|z+\frac{u}{\theta^{*}}-y^{*}\right\|=d\left(z+\frac{u}{\theta^{*}}, C\right) \leq\left\|z+\frac{u}{\theta^{*}}-z\right\|=\left\|\frac{u}{\theta^{*}}\right\|=\frac{1}{\theta^{*}},
$$

where the first equality follows from the first statement in Lemma 2.6. Thus, it follows from (6.21) and (6.22) that

$$
\left\|y-y^{*}\right\|=\theta^{*} \delta\left\|z+\frac{u}{\theta^{*}}-y^{*}\right\| \leq \delta, \quad \text { i.e., } \quad y \in C+\delta B_{H} .
$$

This proves that (6.5) holds with $\gamma_{\rho}$ and $\rho$ being replaced by $\bar{\gamma}_{\rho}$ and $\bar{\rho}<\rho$, respectively. Using the implication (6.5) $\Rightarrow(6.4)$ that was proved above, we obtain that

$$
d(x, C) \leq \bar{\gamma}_{\rho} \max _{1 \leq i \leq m} d\left(x, C_{i}\right) \quad \text { whenever }\|x\| \leq \bar{\rho},
$$

where $\bar{\gamma}_{\rho}>\gamma_{\rho}$ and $\bar{\rho}<\rho$. Letting $\bar{\gamma}_{\rho} \rightarrow \gamma_{\rho}$ and $\bar{\rho} \rightarrow \rho$ in (6.24), and using the continuity of the distance function, we obtain (6.4).

The above proof shows that $(6.4) \Leftrightarrow(6.5) \Rightarrow(6.6) \Rightarrow(6.7) \Rightarrow(6.4)$ and $(6.5) \Rightarrow$ $(\underline{6.8}) \Rightarrow(\underline{6.4})$. This completes the proof of Lemma 6.1. 
From Lemma 6.1 we can easily derive characterizations of (bounded) linear regularity for convex sets. For linear regularity, Lemma 6.1 actually shows that the linear regularity constant (the smallest $\gamma$ that satisfies (6.2) ) is equal to the normality constant of scaling or shifting of $\left\{C_{1}, \ldots, C_{m}\right\}$. Such quantitative relationships between linear regularity and normality are stated next.

Theorem 6.1. Let $C_{1}, \ldots, C_{m}$ be closed convex subsets of $H$ with $C:=\bigcap_{i=1}^{m} C_{i} \neq$ $\emptyset, \gamma>0$, and $\tau>0$. Then the following statements are equivalent:

(1) $d(x, C) \leq \gamma \max _{1 \leq i \leq m} d\left(x, C_{i}\right)$ for all $x \in H$ (Linear Regularity Property);

(2) $\lambda_{N}\left(\theta C_{1}, \ldots, \theta C_{m}\right) \geq \frac{1}{\gamma}$ for all $\theta>0$ (Uniform Normal Property);

(3) $\lambda_{N}\left(\theta C_{1}, \ldots, \theta C_{m}\right) \geq \frac{1}{\gamma}$ for all $\theta \geq \frac{1}{\tau}$;

(4) $\lambda_{N}\left(\operatorname{con}\left(C_{1}-x\right), \ldots, \operatorname{con}\left(C_{m}-x\right)\right) \geq \frac{1}{\gamma}$ for all $x \in C$

(5) $d(x, C) \leq \gamma \max _{1 \leq i \leq m} d\left(x, C_{i}\right)$ for all $x \in H$ with $d(x, C) \leq \frac{\tau}{\gamma}$.

Proof. Note that for any $x \in H$, the normality constant and the linear regularity constant for $\left\{C_{1}, \ldots, C_{m}\right\}$ are the same as those for $\left\{C_{1}-x, \ldots, C_{m}-x\right\}$. Thus, without loss of generality (by replacing the sets $C_{i}$ by $C_{i}-x_{0}$ for some point $x_{0} \in C$ if necessary), we may assume that $0 \in C$.

$(1) \Rightarrow(5)$ This is obvious.

$(5) \Rightarrow(1)$ Since (6.7) holds for $\gamma_{\rho}=\gamma$ and any $\rho>0$, by Lemma 6.1, (6.4) holds for $\gamma_{\rho}=\gamma$ and any $\rho>0$. Thus, (1) holds.

$(1) \Rightarrow(2)$ Since (6.4) holds for $\gamma_{\rho}=\gamma$ and any $\rho>0$, Lemma 6.1 implies that (6.5) holds for $\gamma_{\rho}=\gamma$ and any $\rho>0$, i.e.,

$$
\bigcap_{i=1}^{m}\left(C_{i}+\frac{\delta}{\gamma} B_{H}\right) \subset C+\delta B_{H} \text { for every } \delta>0 .
$$

Dividing both sides of (6.25) by $\delta$ and setting $\theta=1 / \delta$, we get

$$
\bigcap_{i=1}^{m}\left(\theta C_{i}+\frac{1}{\gamma} B_{H}\right) \subset\left(\bigcap_{i=1}^{m} \theta C_{i}\right)+B_{H} \text { for every } \theta>0 .
$$

Using Definition 4.2 for normality constants, (2) follows from (6.26).

$(2) \Rightarrow(4)$ Using Definition 4.2. (2) implies that

$$
\bigcap_{i=1}^{m}\left(\theta C_{i}+\frac{1}{\bar{\gamma}} B_{H}\right) \subset\left(\bigcap_{i=1}^{m} \theta C_{i}\right)+B_{H} \text { for every } \theta>0 \text { and } \bar{\gamma}>\gamma
$$

Since (6.27) implies that (6.5) holds for $\gamma_{\rho}=\bar{\gamma}$ and any $\rho>0$, Lemma 6.1 implies that (6.8) holds for $\gamma_{\rho}=\bar{\gamma}$ and any $\rho>0$. Thus,

$$
\lambda_{N}\left(\operatorname{con}\left(C_{1}-x\right), \ldots, \operatorname{con}\left(C_{m}-x\right)\right) \geq \frac{1}{\bar{\gamma}} \text { for all } x \in C
$$

Letting $\bar{\gamma} \rightarrow \gamma$ in (6.28), we obtain (4).

$(4) \Rightarrow(1)$ Since (6.8) holds for $\gamma_{\rho}=\gamma$ and any $\rho>0$, Lemma 6.1 implies that (6.4) holds for $\gamma_{\rho}=\gamma$ and any $\rho>0$. Therefore (1) holds.

$(2) \Rightarrow(3)$ This is obvious. 
(3) $\Rightarrow$ (1) By Definition 4.2 for any $\bar{\gamma}>\gamma$, we have

$$
\bigcap_{i=1}^{m}\left(\theta C_{i}+\frac{1}{\bar{\gamma}} B_{H}\right) \subset\left(\bigcap_{i=1}^{m} \theta C_{i}\right)+B_{H} \text { for all } \theta \geq \frac{1}{\tau} .
$$

Let $\delta=1 / \theta$. Dividing both sides of $(\underline{6.29}$ by $\theta$ yields

$$
\bigcap_{i=1}^{m}\left(C_{i}+\frac{\delta}{\bar{\gamma}} B_{H}\right) \subset\left(\bigcap_{i=1}^{m} C_{i}\right)+\delta B_{H} \text { for every } 0<\delta \leq \tau .
$$

By (6.30), 6.6) holds for $\gamma_{\rho}=\bar{\gamma}$ and any $\rho>0$. By Lemma 6.1 6.4 holds for $\gamma_{\rho}=\bar{\gamma}$ and any $\bar{\rho}>0$. Since $\bar{\gamma}>\gamma$ is arbitrary, (6.4) holds for $\gamma_{\rho}=\gamma$ and any $\rho>0$, i.e., (1) holds. This completes the proof of Theorem6.1.

Remark 6.2. A more general form of the implication of $(5) \Rightarrow(1)$ was given by Li and Singer [33, Theorem 4] that shows how to establish global error bounds for convex multifunctions by using error bounds for approximate solutions in a small neighborhood of the solution set. In fact, let $\Gamma(t):=\left\{y \mid d\left(y, C_{i}\right) \leq t\right.$ for $1 \leq i \leq m\}$. Then $C=\Gamma(0)$. Applying Theorem 4 in [33] with $x_{0}=0$ and $X=\mathbb{R}$, we get the implication $(5) \Rightarrow(1)$.

To make the quantitative relationship between linear regularity and normality more transparent, we formulate the following corollary of Theorem 6.1.

Corollary 6.1. Let $C_{1}, \ldots, C_{m}$ be closed convex subsets of $H$ with $C:=\bigcap_{1}^{m} C_{i} \neq \emptyset$. Then

$$
\begin{aligned}
\inf _{x \notin C} \frac{\max _{1 \leq i \leq m} d\left(x, C_{i}\right)}{d(x, C)} & =\inf _{x \in C} \lambda_{N}\left(\operatorname{con}\left(C_{1}-x\right), \ldots, \operatorname{con}\left(C_{m}-x\right)\right) \\
& =\inf _{\theta>0} \lambda_{N}\left(\theta C_{1}, \ldots, \theta C_{m}\right)=\inf _{0<\theta \leq \tau} \lambda_{N}\left(\theta C_{1}, \ldots, \theta C_{m}\right),
\end{aligned}
$$

where $\tau>0$ is any positive constant.

Proof. Fix any $\tau>0$ and define the scalars $\lambda_{i}(i=1,2,3,4)$ by

$$
\begin{aligned}
\lambda_{1} & :=\inf _{x \notin C} \frac{\max _{1 \leq i \leq m} d\left(x, C_{i}\right)}{d(x, C)}, \\
\lambda_{2} & :=\inf _{x \in C} \lambda_{N}\left(\operatorname{con}\left(C_{1}-x\right), \ldots, \operatorname{con}\left(C_{m}-x\right)\right), \\
\lambda_{3} & :=\inf _{\theta>0} \lambda_{N}\left(\theta C_{1}, \ldots, \theta C_{m}\right), \\
\lambda_{4} & :=\inf _{0<\theta \leq \tau} \lambda_{N}\left(\theta C_{1}, \ldots, \theta C_{m}\right) .
\end{aligned}
$$

Theorem 6.1 shows that if one $\lambda_{k}$ is positive, then all $\lambda_{k}$ are positive. So we may assume that $\lambda_{k}>0$ for every $k \in\{1,2,3,4\}$. For any such $k$, the definition of $\lambda_{k}$ shows that Theorem 6.1 $\mathrm{k}$ ) must hold for $\gamma=1 / \lambda_{k}$. Thus, by Theorem 6.1

$$
\lambda_{j} \geq \frac{1}{\gamma}=\frac{1}{\left(1 / \lambda_{k}\right)}=\lambda_{k} \text { for all } 1 \leq j \leq 4
$$

Since $1 \leq k \leq 4$ was arbitrary, we obtain that $\lambda_{1}=\lambda_{2}=\lambda_{3}=\lambda_{4}$. This proves Corollary 6.1. 
Remark 6.3. Note that $\left\{\theta C_{1}, \ldots, \theta C_{m}\right\}$ is a scaling of the original collection of convex sets, and

$$
\operatorname{con}\left(C_{i}-x\right)=\bigcup_{\theta>0} \theta\left(C_{i}-x\right)
$$

is the union of shifting (subtracting by $x$ ) and scaling (multiplying by $\theta$ ) of $C_{i}$ with respect to $\theta>0$. The above corollary shows how the linear regularity constant is related to the normality constants of families of convex sets generated by shifting and scaling of $\left\{C_{1}, \ldots, C_{m}\right\}$.

The above corollary clearly describes the quantitative relationship between the linear regularity and the normal property, but it is less transparent about the qualitative relationship between linear regularity and normality. To remedy this, we include various characterizations of linear regularity in the following theorem.

Theorem 6.2. Let $C_{1}, \ldots, C_{m}$ be closed convex subsets of $H$ with $C:=\bigcap_{1}^{m} C_{i} \neq \emptyset$. Then the following statements are equivalent:

(1) $\left\{C_{1}, \ldots, C_{m}\right\}$ has the linear regularity property;

(2) $\left\{C_{1}, \ldots, C_{m}\right\}$ has the uniform normal property;

(3) There exist positive constants $\tau$ and $\gamma$ such that

$$
\bigcap_{1}^{m}\left(C_{i}+\frac{\delta}{\gamma} B_{H}\right) \subset C+\delta B_{H} \text { for all } 0<\delta \leq \tau ;
$$

(4) There exists a positive constant $\gamma$ such that

$$
\bigcap_{1}^{m}\left(\operatorname{con}\left(C_{i}-x\right)+\frac{1}{\gamma} B_{H}\right) \subset \operatorname{con}(C-x)+B_{H} \text { for all } x \in C ;
$$

(5) $\left\{C_{1}, \ldots, C_{m}\right\}$ has the CHIP, and there exists a positive constant $\hat{\gamma}$ such that

$$
B_{H} \cap\left(\sum_{i=1}^{m}\left(C_{i}-x\right)^{\ominus}\right) \subset \sum_{i=1}^{m}\left(\frac{1}{\hat{\gamma}} B_{H}\right) \cap\left(C_{i}-x\right)^{\ominus} \text { for all } x \in C .
$$

Proof. The equivalence of (1)-(4) follows from Theorem 6.1 It suffices to prove the equivalence of (4) and (5).

First assume that (5) holds. Note $\left(C_{i}-x\right)^{\ominus}=\left[\operatorname{con}\left(C_{i}-x\right)\right]^{\circ}$ and the strong CHIP implies the closed intersection property of $\left\{\operatorname{con}\left(C_{1}-x\right), \ldots, \operatorname{con}\left(C_{m}-x\right)\right\}$ (see Theorem 5.1). If (6.33) holds, then Theorems 4.2 and 4.3 imply that

$$
\begin{aligned}
\frac{1}{\hat{\gamma}} & \leq \lambda_{G}\left(\left[\operatorname{con}\left(C_{1}-x\right)\right]^{\circ}, \ldots,\left[\operatorname{con}\left(C_{m}-x\right)\right]^{\circ}\right) \\
& \leq m \cdot \lambda_{D}\left(\left[\operatorname{con}\left(C_{1}-x\right)\right]^{\circ}, \ldots,\left[\operatorname{con}\left(C_{m}-x\right)\right]^{\circ}\right) \\
& =m \cdot \lambda_{N}\left(\operatorname{con}\left(C_{1}-x\right), \ldots, \operatorname{con}\left(C_{m}-x\right)\right) \text { for all } x \in C,
\end{aligned}
$$

which implies that (4) holds with any $\gamma>m \hat{\gamma}$.

On the other hand, if (6.32) holds, then Theorem [3.1(4) implies that $\left\{\operatorname{con}\left(C_{1}-x\right), \ldots, \operatorname{con}\left(C_{m}-x\right)\right\}$ has the closed intersection property. Using 
Theorems 4.2 and 4.3 we obtain that

$$
\begin{aligned}
\frac{1}{\gamma} & \leq \lambda_{N}\left(\operatorname{con}\left(C_{1}-x\right), \ldots, \operatorname{con}\left(C_{m}-x\right)\right) \\
& =\lambda_{D}\left(\left[\operatorname{con}\left(C_{1}-x\right)\right]^{\circ}, \ldots,\left[\operatorname{con}\left(C_{m}-x\right)\right]^{\circ}\right) \\
& =\lambda_{D}\left(\left(C_{1}-x\right)^{\ominus}, \ldots,\left(C_{m}-x\right)^{\ominus}\right) \\
& \leq \lambda_{G}\left(\left(C_{1}-x\right)^{\ominus}, \ldots,\left(C_{m}-x\right)^{\ominus}\right) \text { for all } x \in C .
\end{aligned}
$$

Thus (5) holds for $\hat{\gamma}>\gamma$. This completes the proof of Theorem 6.2

Remark 6.4. The implication $(5) \Rightarrow(1)$ in Theorem 6.2 was proved earlier in 9 , Theorem 4.2].

Similarly, from Lemma 6.1 we can easily derive various characterizations of bounded linear regularity for convex sets.

Theorem 6.3. Let $C_{1}, \ldots, C_{m}$ be closed convex subsets of $H$ with $C:=\bigcap_{1}^{m} C_{i} \neq \emptyset$. Then the following statements are equivalent:

(1) $\left\{C_{1}, \ldots, C_{m}\right\}$ has the bounded linear regularity property;

(2) For any $\rho>0$, there exists $\gamma_{\rho}>0$ such that

$$
\left(\rho B_{H}\right) \cap\left[\bigcap_{1}^{m}\left(C_{i}+\frac{\delta}{\gamma_{\rho}} B_{H}\right)\right] \subset C+\delta B_{H} \text { for all } \delta>0 ;
$$

(3) Let $\tau>0$ be a fixed positive constant. For any $\rho>0$, there exists $\gamma_{\rho}>0$ such that

$$
\left(\rho B_{H}\right) \cap\left[\bigcap_{1}^{m}\left(C_{i}+\frac{\delta}{\gamma_{\rho}} B_{H}\right)\right] \subset C+\delta B_{H} \text { for all } 0<\delta \leq \tau ;
$$

(4) For any $\rho>0$, there exists $\gamma_{\rho}>0$ such that

$$
\bigcap_{1}^{m}\left(\operatorname{con}\left(C_{i}-x\right)+\frac{1}{\gamma_{\rho}} B_{H}\right) \subset \operatorname{con}(C-x)+B_{H} \text { for all } x \in C \text { with }\|x\| \leq \rho ;
$$

(5) $\left\{C_{1}, \ldots, C_{m}\right\}$ has CHIP, and for any $\rho>0$, there exists $\gamma_{\rho}>0$ such that

$$
B_{H} \cap\left(\sum_{i=1}^{m}\left(C_{i}-x\right)^{\ominus}\right) \subset \sum_{i=1}^{m}\left(\frac{1}{\hat{\gamma}_{\rho}} B_{H}\right) \cap\left(C_{i}-x\right)^{\ominus} \text { for all } x \in C \text { with }\|x\| \leq \rho .
$$

Proof. The equivalence of (1)-(4) follows from Lemma 6.1. Replacing $x \in C$ by $x \in C \cap\left(\rho B_{H}\right), \gamma$ by $\gamma_{\rho}$, and $\hat{\gamma}$ by $\hat{\gamma}_{\rho}$ in the proof of Theorem [6.2, we get a proof of the equivalence of (4) and (5) in Theorem 6.3.

Finally, when applied to closed convex cones, we obtain the following characterizations of linear regularity. The equivalence of the first four statements in the following corollary are known (see [26, Theorem 2.1] and [9, Theorem 6.5]).

Corollary 6.2. Let $K_{1}, \ldots, K_{m}$ be closed convex cones in $H$. Then the following statements are equivalent:

(1) $\left\{K_{1}, \ldots, K_{m}\right\}$ is boundedly linearly regular; 
(2) $\left\{K_{1}, \ldots, K_{m}\right\}$ is linearly regular;

(3) $\left\{K_{1}, \ldots, K_{m}\right\}$ has the normal property;

(4) $\left\{K_{1}^{\circ}, \ldots, K_{m}^{\circ}\right\}$ has property $(\mathrm{G})$;

(5) $\left\{K_{1}^{\circ}, \ldots, K_{m}^{\circ}\right\}$ has the dual normal property;

(6) $\left\{K_{1}, \ldots, K_{m}\right\}$ has the uniform normal property.

Proof. The equivalence of (1) and (2) was proved earlier in [4, Theorem 6.5]. By Theorem 6.2 $\left\{K_{1}, \ldots, K_{m}\right\}$ is linearly regular if and only if it has the uniform normal property. Thus (2) and (6) are equivalent. By Theorem 3.1(3), the uniform normal property is equivalent to the normal property, so (3) and (6) are equivalent. Since $K_{i}$ are closed, $\left\{K_{1}, \ldots, K_{m}\right\}$ has the closed intersection property. By Corollary 4.1, (3) and (4) are equivalent. Finally, (3) and (5) are equivalent by Theorem 4.2. This completes the proof of Corollary 6.2

\section{REFERENCES}

[1] A. Bakan, Normal pairs of cones in finite-dimensional spaces, in "Questions of the Theory of Approximation of Functions and Their Applications," Inst. Mat., Akad. Nauk Ukr. SSR, Kiev, 1988 (in Russian). MR90h:52001

[2] A. Bakan, The Moreau-Rockafellar equality for sublinear functionals, Transl. Ukr. Math. J., 41(1990), 861-871. MR:1019523 (91a:58025)

[3] A. Bakan, Nonemptiness of classes of normal pairs of cones of transfinite order, Transl. Ukr. Math. J., 41(1989), 462-466. MR1004860 (90g:52001)

[4] H. H. Bauschke, Projection Algorithms and Monotone Operators, Ph.D. thesis, Simon Fraser University, 1996.

[5] H. Bauschke and J. Borwein, On the convergence of von Neumann's alternating projection algorithm for two sets, Set-Valued Analysis, 1(2)(1993), 185-212. MR.1239403 (95d:65048)

[6] H. Bauschke and J. Borwein, On projection algorithms for solving convex feasibility problems, SIAM Review, 38(1996), 367-426. MR1409591 (98f:90045)

[7] H. Bauschke and J. Borwein, Conical open mapping theorems and regularity, Proceedings of the Centre for Mathematics and its Applications, 36 (Australian National University, 1998), $1999,1-10$.

[8] H. Bauschke, J. Borwein, and A. Lewis, The method of cyclic projections for closed convex sets in Hilbert space, in Optimization and Nonlinear Analysis (edited by Y. Censor and S. Reich), Contemporary Mathematics, Amer. Math. Soc., 1997. MR1442992 (98c:49069)

[9] H. Bauschke, J. Borwein, and W. Li, Strong conical hull intersection property, bounded linear regularity, Jameson's property $(\mathrm{G})$, and error bounds in convex optimization, Mathematical Programming (Series A), 86(1)(1999), 135-160. MR1712477 (2000f:90095)

[10] H. Bauschke, J. Borwein, and P. Tseng, Bounded linear regularity, strong CHIP, and CHIP are distinct properties, J. Convex Anal., 7(2000), 395-412. MR.1811687(2002d:90113)

[11] C. Chui, F. Deutsch, and J. Ward, Constrained best approximation in Hilbert space, Constructive Approx., 6(1990), 35-64. MR,1027508 (91b:41014)

[12] C.K. Chui, F. Deutsch, and J.D. Ward, Constrained best approximation in Hilbert space II, J. Approx. Theory, 71(1992), 231-238. MR.1186970 (93k:41019)

[13] S. Deng, Perturbation analysis of a condition number for convex inequality systems and global error bounds for analytic systems, Math. Programming, 83(1998), 263-276. MR1647861 (99j:90094)

[14] S. Deng, Global error bounds for convex inequalities in Banach spaces, SIAM J. Control Optimiz., 36(4)(1998), 1240-1249. MR1618049|(99f:90142)

[15] F. Deutsch, The role of the strong conical hull intersection property in convex optimization and approximation, in Approximation Theory IX, Vol. I: Theoretical Aspects (edited by C.K. Chui and L.L. Schumaker), Vanderbilt University Press, Nashville, TN, 1998, pp. 105112. MR:1742997|(2001c:90098)

[16] F. Deutsch, Best Approximation in Inner Product Spaces, Springer, New York, 2001. MR.1823556(2002c:41001)

[17] F. Deutsch, W. Li, and J. Swetits, Fenchel duality and the strong conical hull intersection property, J.Optimiz. Theory Appl., 102(1999), 681-695. MR.1710727(2000k:49047) 
[18] F. Deutsch, W. Li, and J. Ward, A dual approach to constrained interpolation from a convex subset of Hilbert space, J. Approx. Theory, 90(1997), 385-414. MR.1469335 (98m:41050)

[19] F. Deutsch, W. Li, and J.D. Ward, Best approximation from the intersection of a closed convex set and a polyhedron in Hilbert space, weak Slater conditions, and the strong conical hull intersection property, SIAM J. Optimiz., 10(2000), 252-268. MR.1742319|(2001a:41047)

[20] J. Diestel, Geometry of Banach spaces, Selected Topics, Lect. Not. in Math., Springer-Verlag, 1980. MR0461094 (57:1079)

[21] C. Franchetti and W. Light, The alternating algorithm in uniformly convex spaces, J. London Math. Soc., 29(1984), 545-555. MR0754940 (85h:41064)

[22] J.-B. Hiriart-Urruty and C. Lemarechal, Convex analysis and minimization algorithms I, Springer-Verlag, N.Y., 1993.

[23] A. Hoffman, On approximate solutions of systems of linear inequalities. J. Res. Natl. Bur. Standards, 49(1952), 263-265. MR0051275 (14:455b)

[24] A. Ioffe and V. Tikhomirov, Theory of Extremal Problems, Nauka, Moscow, 1974 (in Russian) [English translation by North Holland, Amsterdam, 1979].

[25] D. Klatte and W. Li, Asymptotic constraint qualifications and global error bounds for convex inequalities, Math. Programming, 84(1999), 137-160. MR 1687264|(2000f:90073)

[26] G. Jameson, The duality of pairs of wedges, Proc. London Math. Soc., 24(1972), 531-547. MR 0298388(45:7440)

[27] A. Kusraev, Vector Duality and Its Application (in Russian), Nauka, Novosybirsk, 1985. MR0836135 (87f:46014)

[28] A. Kusraev and S. Kutateladze, Subdifferential Calculus, Nauka, Novosybirsk, 1987 (in Russian) [English translation as: Subdifferentials: Theory and Applications, Mathematics and its Applications, Vol. 323, Kluwer Academic Publ., 1995]. MR.1471481 (99c:46001)

[29] P.-J.Laurent, Approximation et Optimization, Univ. Sci. Med. Grenoble, Hermann, Paris, 1972. MR0467080 (57:6947)

[30] A. S. Lewis and J.-S. Pang, Error bounds for convex inequality systems, in Generalized Convexity, Generalized Monotonicity (edited by J.-P. Crouzeix, J.-E. Martizez-Legaz, and M. Volle), 1998, pp. 75-110. MR.1646951 (2000d:90082)

[31] W. Li, Abadie's constraint qualification, metric regularity, and error bounds for differentiable convex inequalities, SIAM J. Optim., 7(1997), 966-978. MR1479609 (99b:90131)

[32] W. Li, C. Nahak, and I. Singer, Constraint qualifications for semi-infinite systems of convex inequalities, SIAM J. Optimiz., 11(2000), 31-52. MR.1785387|(2001i:90096)

[33] W. Li and I. Singer, Global error bounds for convex multifunctions and applications, Math. Oper. Res., 23(1998), 443-462. MR.1626694(99i:90097)

[34] R. Rockafellar, Convex Analysis, Princeton Univ. Press, Princeton, N.J., 1970. MR0274683 $(43: 445)$

[35] H. Schaefer, Topological Vector Spaces, Macmillan, N.Y., 1966. MR0193469 (33:1689)

Institute of Mathematics, National Academy of Sciences of Ukraine, Kyiv 01601, UKRAINE

E-mail address: andrew@bakan.kiev.ua

Department of Mathematics, The Pennsylvania State University, University Park, PENNSYlVANia 16802

E-mail address: deutsch@math.psu.edu

NASA Langley Research Center, Hampton, Virginia 23681

E-mail address: w.li@nasa.gov 